\title{
RADSAT Benchmarks for Prompt Gamma Neutron Activation Analysis Measurements
}

\author{
KA Burns \\ CJ Gesh
}

July 2011

\section{Pacific Northwest}

NATIONAL LABORATORY 


\title{
DISCLAIMER
}

This report was prepared as an account of work sponsored by an agency of the United States Government. Neither the United States Government nor any agency thereof, nor Battelle Memorial Institute, nor any of their employees, makes any warranty, express or implied, or assumes any legal liability or responsibility for the accuracy, completeness, or usefulness of any information, apparatus, product, or process disclosed, or represents that its use would not infringe privately owned rights. Reference herein to any specific commercial product, process, or service by trade name, trademark, manufacturer, or otherwise does not necessarily constitute or imply its endorsement, recommendation, or favoring by the United States Government or any agency thereof, or Battelle Memorial Institute. The views and opinions of authors expressed herein do not necessarily state or reflect those of the United States Government or any agency thereof.

\author{
PACIFIC NORTHWEST NATIONAL LABORATORY \\ operated by \\ BATTELLE \\ for the \\ UNITED STATES DEPARTMENT OF ENERGY \\ under Contract DE-AC05-76RL01830
}

Printed in the United States of America

Available to DOE and DOE contractors from the

Office of Scientific and Technical Information,

P.O. Box 62, Oak Ridge, TN 37831-0062;

ph: (865) 576-8401

fax: $(865) 576-5728$

email: reports@adonis.osti.gov

\footnotetext{
Available to the public from the National Technical Information Service, U.S. Department of Commerce, 5285 Port Royal Rd., Springfield, VA 22161 ph: (800) 553-6847 fax: $(703) 605-6900$

email: orders@ntis.fedworld.gov

online ordering: http://www.ntis.gov/ordering.htm
}

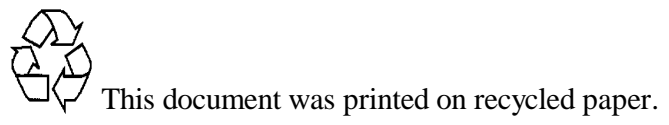




\section{RADSAT Benchmarks for Prompt Gamma Neutron Activation Analysis Measurements}

KA Burns

CJ Gesh

July 2011

Prepared for the U. S. DEPARTMENT OF ENERGY

under Contract DE-AC05-76RL01830

Pacific Northwest National Laboratory

Richland, WA 99354 


\section{Summary}

The accurate and efficient simulation of coupled neutron-photon problems is necessary for several important radiation detection applications. Examples include the detection of nuclear threats concealed in cargo containers and prompt gamma neutron activation analysis for nondestructive determination of elemental composition of unknown samples. High-resolution gamma-ray spectrometers are used in these applications to measure the spectrum of the emitted photon flux, which consists of both continuum and characteristic gamma rays with discrete energies. Monte Carlo transport is the most commonly used simulation tool for this type of problem, but computational times can be prohibitively long. This work explores the use of multi-group deterministic methods for the simulation of coupled neutron-photon problems.

The main purpose of this work is to benchmark several problems modeled with RADSAT and MCNP to experimental data. Additionally, the cross section libraries for RADSAT are updated to include ENDF/BVII cross sections. Preliminary findings show promising results when compared to MCNP and experimental data, but also areas where additional inquiry and testing are needed. The potential benefits and shortcomings of the multi-group-based approach are discussed in terms of accuracy and computational efficiency.

Key Words: Coupled Neutron-Photon Transport, Neutron Activation Analysis, Multi-Group Cross sections, Neutron Photon Problems 


\section{Contents}

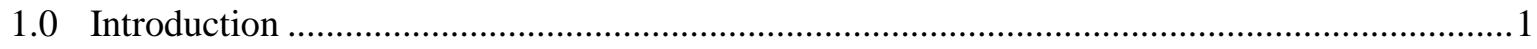

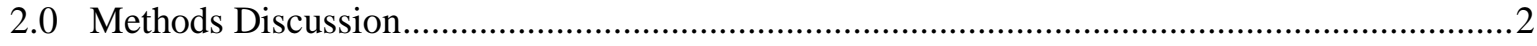

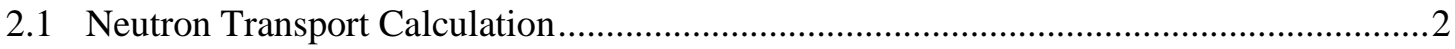

2.2 RADSAT-NG Neutron Gamma Cross Section Library Preparation...................................3

2.3 Induced Photon Spectrum Generation............................................................................

2.4 Photon Transport Calculation.................................................................................

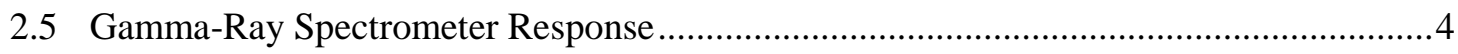

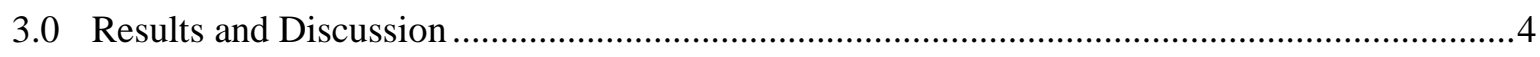

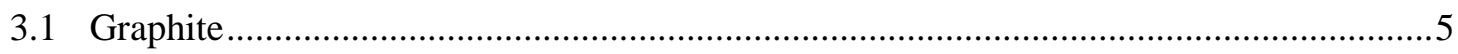

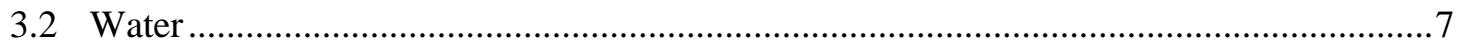

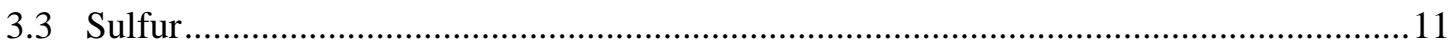

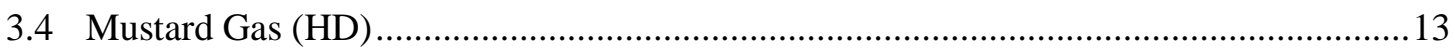

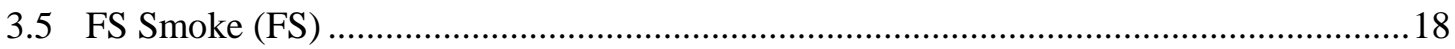

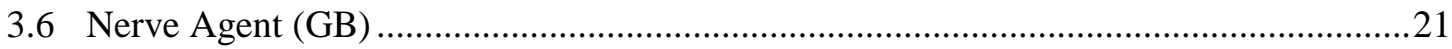

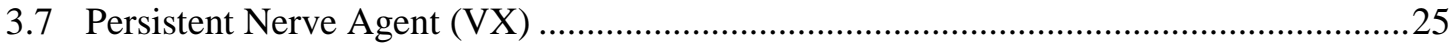

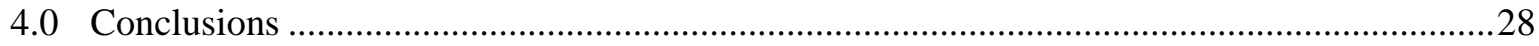

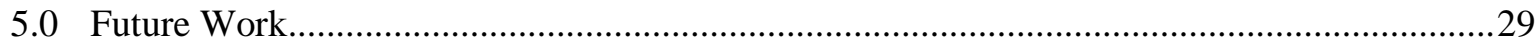

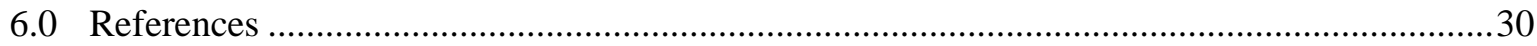




\section{Figures}

Figure 1. Procedure for Calculating Coupled Neutron-Photon Problems using RADSAT ................2

Figure 2. PINS Configuration used in RADSAT Modeling .........................................................5

Figure 3. Empirical data from the irradiation of graphite blocks (Data provided by INL)...............6

Figure 4. Comparison of MCNP and RADSAT detector response for graphite irradiated in PINS

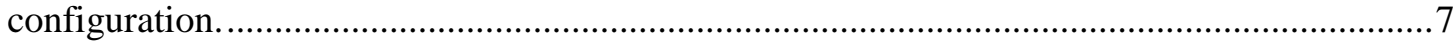

Figure 5. Empirical data from the irradiation of a 1-Liter water bottle (Data provided by INL). ......8

Figure 6. Empirical data from the irradiation of a 4-Liter water bottle (Data provided by INL).......9

Figure 7. Comparison of MCNP and RADSAT detector response for 1-Liter water bottle

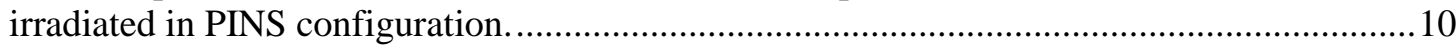

Figure 8. Comparison of MCNP and RADSAT detector response for 4-Liter water bottle

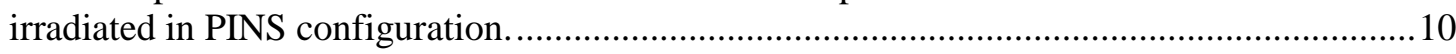

Figure 9. Empirical data from the irradiation of flaked sulfur (Data provided by INL)................12

Figure 10. Comparison of MCNP and RADSAT detector response for sulfur irradiated in PINS

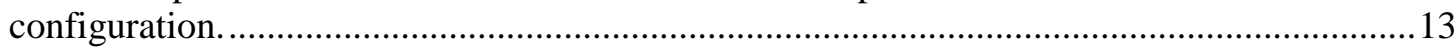

Figure 11. Empirical data from the irradiation of HD (Data provided by INL). ...........................14

Figure 12. Comparison of MCNP and RADSAT detector response for HD irradiated in PINS

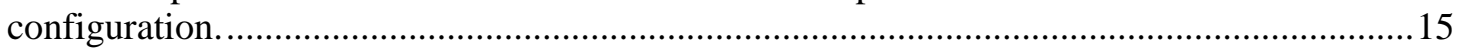

Figure 13. Comparison of 1.95 and $1.959 \mathrm{MeV}$ chlorine doublet in MCNP and RADSAT detector response for HD irradiated in PINS configuration...........................................................16

Figure 14. Comparison of 2.223 MeV hydrogen and 2.23 MeV sulfur peaks in MCNP and RADSAT detector response for HD irradiated in PINS configuration....................................16

Figure 15. Comparison of 5.421 MeV sulfur peak in MCNP and RADSAT detector response for HD irradiated in PINS configuration.

Figure 16. Comparison of 7.631 MeV and 7.645 MeV iron doublet in MCNP and RADSAT detector response for HD irradiated in PINS configuration...................................................17

Figure 17. Empirical data from the irradiation of FS (Data provided by INL)............................18

Figure 18. Comparison of MCNP and RADSAT detector response for FS irradiated in PINS configuration.

Figure 19. Comparison of 1.95 and $1.959 \mathrm{MeV}$ chlorine doublet in MCNP and RADSAT detector response for FS irradiated in PINS configuration..........................................................20 
Figure 20. Comparison of 2.223 MeV hydrogen and 2.23 MeV sulfur peaks in MCNP and RADSAT detector response for FS irradiated in PINS configuration.

Figure 22. Comparison of 7.631 MeV and 7.645 MeV iron doublet in MCNP and RADSAT detector response for FS irradiated in PINS configuration. ...................................................21

Figure 23. Empirical data from the irradiation of GB (Data provided by INL).............................22

Figure 24. Comparison of MCNP and RADSAT detector response for GB irradiated in PINS configuration.

Figure 25. Comparison of $1.266 \mathrm{MeV}$ phosphorus peak in MCNP and RADSAT detector response for GB irradiated in PINS configuration..

Figure 26. Comparison of 2.223 MeV hydrogen and 2.233 MeV phosphorus peaks in MCNP and RADSAT detector response for GB irradiated in PINS configuration.

Figure 26. Comparison of 7.631 MeV and 7.645 MeV iron doublet in MCNP and RADSAT detector response for GB irradiated in PINS configuration..................................................24

Figure 27. Empirical data from the irradiation of VX (Data provided by INL).

Figure 28. Comparison of MCNP and RADSAT detector response for VX irradiated in PINS

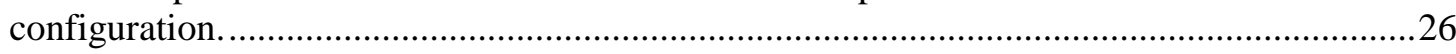

Figure 29. Comparison of $1.266 \mathrm{MeV}$ phosphorus peak in MCNP and RADSAT detector response for VX irradiated in PINS configuration..............................................................26

Figure 31. Comparison of 2.223 MeV hydrogen, 2.23 MeV sulfur, and 2.233 MeV phosphorus peaks in MCNP and RADSAT detector response for VX irradiated in PINS configuration....27

Figure 32. Comparison of 5.421 MeV sulfur peak in MCNP and RADSAT detector response for VX irradiated in PINS configuration.

Figure 32. Comparison of 7.631 MeV and 7.645 MeV iron doublet in MCNP and RADSAT detector response for VX irradiated in PINS configuration. .28 


\section{Tables}

Table 1. Peak count rates for regions of interest from the irradiation of HD..................................14

Table 2. Comparison of peak ratio for irradiation of HD. ............................................................15

Table 3. Peak count rates for regions of interest from the irradiation of FS..................................19

Table 4. Comparison of peak ratio for irradiation of FS............................................................. 19

Table 5. Peak count rates for regions of interest from the irradiation of GB...............................22

Table 5. Peak count rates for regions of interest from the irradiation of VX.................................25 


\section{Acronyms}

CEPXS Coupled Electron-Photon Cross Section

ENDF Evaluated Nuclear Data File

FS FS Smoke

GB Nerve Agent (Sarin)

$\mathrm{HCl} \quad$ Hydrogen Chloride

HD Sulfur Mustard Gas

HPGe High-purity Germanium

INL Idaho National Laboratory

MCNP Monte Carlo for Neutrons and Photons

PGNAA Prompt Gamma Neutron Activation Analysis

PINS Portable Isotopic Neutron Spectroscopy

PNNL Pacific Northwest National Laboratory

RADSAT Radiation Detection Scenario Analysis Toolbox

RADSAT-NG Radiation Detection Scenario Analysis Toolbox - Neutron Gamma

VX Persistent Nerve Agent

Page ix of ix 


\subsection{Introduction}

Neutron-induced photon signatures are an important component of many radiation detection and nondestructive assay scenarios. One example is the screening of vehicles and cargo at border crossings wherein neutron-emitting threat sources (e.g., plutonium) can induce photon emissions in the surrounding cargo. Another familiar example is prompt gamma neutron activation analysis (PGNAA), a technique used to determine the composition of unknown samples. In PGNAA, the sample is irradiated with a neutron source and the subsequent interactions in the material produce photons through a number of reactions, including inelastic scatter and radiative capture. The discrete-energy emissions represent unique fingerprints of isotopes in the sample. For example, the $2.223 \mathrm{MeV}$ gamma ray produced from radiative capture in ${ }^{1} \mathrm{H}$, where the presence and relative portions of these discrete gamma rays can be used to infer the isotopic composition of the material being interrogated. A high-resolution gamma-ray spectrometer must be used to obtain the level of detail required to detect and quantify the key characteristic gamma rays, and thus determine composition.

At present, Monte Carlo is the primary tool for simulating neutron activation analysis problems, largely because it is able to preserve the important discrete-energy signatures throughout the problem. This culminates in a pulse-height tally in the high-resolution gamma-ray spectrometer. However, neutronphoton problems analyzed with Monte Carlo for Neutrons and Photons (MCNP) often require very long run times due to the high degree of scatter and absorption during neutron and photon transport.

Multi-group deterministic methods offer potential advantages for neutron-photon problems. Once convergence has reached a global solution, an "infinite real measurement time" solution is obtained. Additionally, deterministic methods offer the ability to reduce computational times, most noticeably in problems with highly scattering or highly absorbing materials. The purpose of this research is to explore the use of multi-group deterministic methods for the simulation of coupled neutron-photon problems.

This work uses the Radiation Detection Scenario Analysis Toolbox (RADSAT) [1][3], which couples the deterministic method and Monte Carlo transport method to perform analysis in three dimensions.

RADSAT uses the deterministic transport code to determine the field solution then calculates the detector response using a Monte Carlo calculation. RADSAT is a collaboration between Pacific Northwest National Laboratory (PNNL) and Transpire, Inc. The deterministic core of RADSAT is Attila ${ }^{\circledR}$, a code originally developed by Los Alamos National Laboratory, and since expanded and refined by Transpire, Inc. [2]. Attila ${ }^{\circledR}$ solves the linearized Boltzmann Transport Equation on unstructured tetrahedral meshes and incorporates ray-effect mitigation techniques well suited to radiation detection problems. MCNP5 [6] is used for the calculation of detector response. Previous publications have described the use of RADSAT for photon-only transport problems [1][3], including the use of an adaptive energy group structure that preserves the discrete nature of characteristic gamma-ray emissions.

A major challenge in using multi-group transport methods for coupled neutron-photon problems is calculating the neutron-induced photon signatures throughout the problem. The preparation of suitable multi-group cross sections is required in order to complete this task. Previous publications have described the standard method for producing multi-group cross sections [4][5].

In this report, the benchmark cases for modeling PGNAA problems using RADSAT are examined, along with its shortcomings for the simulation of high-resolution neutron activation analysis. Additional work that is needed to realize the potential of coupled deterministic/Monte Carlo approaches for neutron activation analysis is outlined in Section 5 of this report. 


\subsection{Methods Discussion}

The RADSAT approach for simulating coupled neutron-photon problems consists of four steps:

1. A multi-group deterministic neutron transport calculation of the source neutrons to all parts of the problem [2].

2. Generation of the corresponding spatially dependent neutron-induced gamma-ray spectrum using the RADSAT-NG cross section library [4][5].

3. A multi-group, deterministic photon transport calculation of the induced gamma-ray spectrum to all parts of the problem [1][3].

4. Calculation of the detector response (i.e., pulse-height tally) in a spectrometer using MCNP5 within RADSAT [1][3].

The process is outlined in Figure 1, where the yellow boxes show RADSAT-NG cross section preparation and neutron-induced photon source generation using RADSAT.

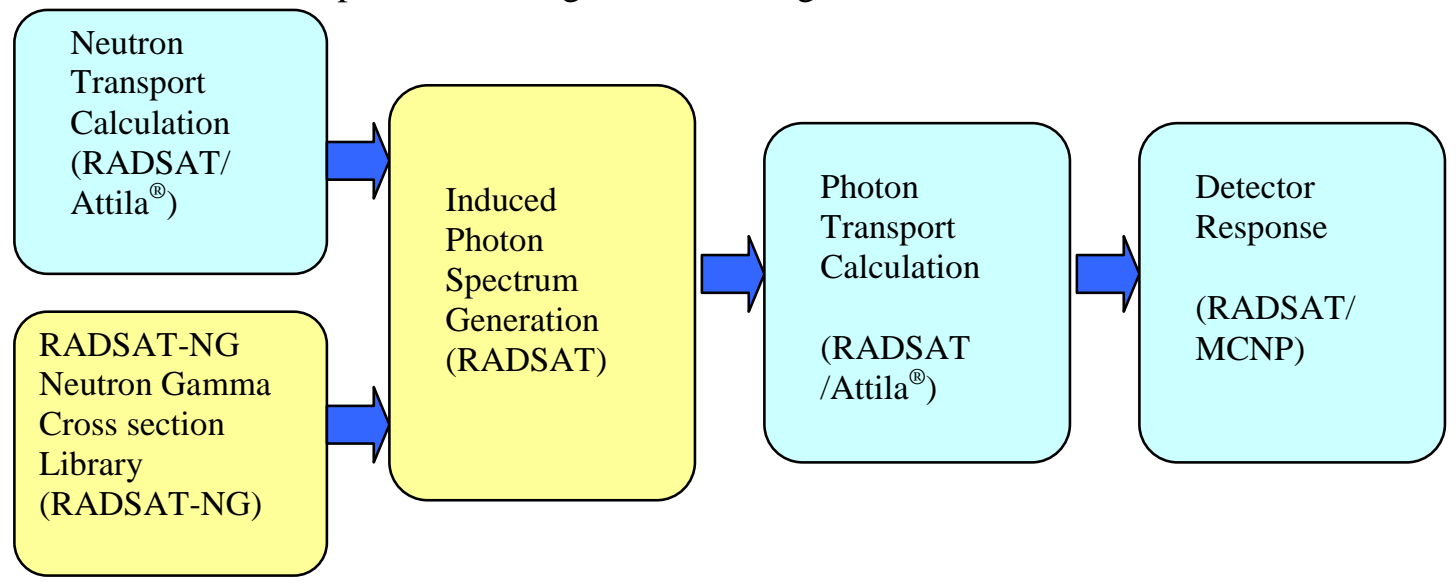

Figure 1. Procedure for Calculating Coupled Neutron-Photon Problems using RADSAT

\subsection{Neutron Transport Calculation}

Neutron transport calculations, completed with Attila ${ }^{\circledR}$ [2], the deterministic backbone of RADSAT were run (unless otherwise noted) with $\mathrm{S}_{8}$ Triangular Chebychev Legendre angular quadrature set, $\mathrm{P}_{2}$ scattering, and the Kynea3 cross section set [7]. The Kynea3 cross section set (developed by Sandia National Laboratories and Oak Ridge National Laboratory) is a general-purpose library for radiation detection scenarios, and includes 36 energy groups below $5 \mathrm{eV}, 25$ energy groups from $5 \mathrm{eV}$ to $1 \mathrm{MeV}$, and 18 energy groups above $1 \mathrm{MeV}$ [7]. The Kynea3 group boundaries provide a balance between thermal and high-energy groups that have been shown effective in neutron activation analysis problems by Sandia National Laboratories; that experience is leveraged as the starting point for this work but further refinements of the neutron energy groups could prove to be beneficial in the future. Evaluated Nuclear Data File B (ENDF/B) was the source of tabulated cross section data for all modeling (both MCNP and RADSAT) in this work. Unless otherwise specified, Evaluated Nuclear Data File ENDF/B version VII was used. 


\subsection{RADSAT-NG Neutron Gamma Cross Section Library Preparation}

Existing multi-group neutron-gamma cross section libraries, like those provided in the Attila ${ }^{\circledR}$ distribution, are more appropriate for photon dose rate and total flux calculations rather than highresolution gamma-ray spectroscopy. Consequently, existing cross section libraries generally have relatively few (e.g., 10 to 20) broad photon groups spanning energies up to several $\mathrm{MeV}$. They are unsuitable for the simulation of high-resolution gamma-ray spectroscopy that requires the preservation of discrete-energy photons. In addition, the existing coupled neutron-gamma cross section libraries combine the cross sections for discrete and continuum gamma productions into one multi-group gamma production matrix. This means that the production rate for the characteristic discrete-energy emissions-the key fingerprints in neutron activation analysis - cannot be calculated separately from the continuum. Instead, the discrete gamma-ray production rates are homogenized into broad energy groups with the continuum.

RADSAT-NG cross sections were developed using a hybrid method starting at the raw ENDF cross section files, using various NJOY subroutines to process the cross sections, then partitioned into a usable format using a PNNL developed code [4][5].

\subsection{Induced Photon Spectrum Generation}

The calculation of the spatially dependent neutron-induced photon spectrum was completed by determining the photon production rate as a function of position and gamma-ray energy. The photon production rate $R\left(E_{\gamma}, \vec{r}\right)$ was calculated separately for each discrete gamma ray using Equation 1 .

$$
R\left(E_{\gamma}, \vec{r}\right)=\sum_{g^{\prime}=1}^{G} N_{i} \sigma_{\left(n, \gamma_{-} \text {discrete }\right)}^{i}\left(E_{g^{\prime}}, E_{\gamma}\right) \phi_{n}^{g^{\prime}}(\vec{r})
$$

where $\mathrm{N}_{\mathrm{i}}$ is the number density for the $\mathrm{i}^{\text {th }}$ isotope, $\phi_{n}^{g}(\vec{r})$ is the spatially dependent neutron flux for energy group g, and $\sigma_{\left(n, \gamma_{\_} \text {discrete }\right)}^{i}\left(E_{g^{\prime}}, E_{\gamma}\right)$ is the photon production cross section for a discrete gamma ray with energy $\mathrm{E}_{\gamma}$ produced by the $\mathrm{i}^{\text {th }}$ isotope. The spatially dependent neutron flux, $\phi_{n}^{g^{\prime}}(\vec{r})$, was obtained from volume-averaged neutron flux edits in Attila ${ }^{\circledR}$.

The RADSAT-NG cross sections were then used for the gamma production cross section. This reaction rate, and therefore photon production rate, was calculated on a volume-by-volume basis for each of the $i$ isotopes included in the Attila ${ }^{\circledR}$ model. Various volume-partitioning schemes have been used to explore the effect of the neutron flux spatial variation on the fidelity of the photon production rate and energy spectrum.

The continuum gamma rays were calculated using a similar method as that used to calculate the discrete gamma rays. The major difference in the two methods is that the continuum was summed over all of the isotopes and/or elements in the model to obtain the total continuum production rate within a specific photon energy group and spatial region using Equation 2.

$$
R\left(E_{g}, \vec{r}\right)=\sum_{i} \sum_{g^{\prime}=1}^{G} N_{i} \sigma_{\left(n, \gamma_{-} \text {continuum }\right)}^{i}\left(E_{g^{\prime}}, E_{g}\right) \phi_{n}^{g^{\prime}}(\vec{r})
$$


where $\sigma_{\left(n, \gamma_{-} \text {continuum }\right)}^{i}\left(E_{g^{\prime}}, E_{g}\right)$ is the photon production cross section for a continuum gamma ray in energy group $\mathrm{E}_{\mathrm{g}}$ produced by the $\mathrm{i}^{\text {th }}$ isotope.

\subsection{Photon Transport Calculation}

The energy- and position-dependent neutron-induced photon source term calculated in Section 2.3 was then used as the photon source term in a photon transport calculation in RADSAT. The photon energy group structure for the gamma model is defined using an adaptive approach described in detail in [1]. To preserve the important gamma-ray lines, narrow “peak" groups $(0.3 \mathrm{keV})$ were centered around the most prominent emission energies in the problem. All lower-yield discrete emissions and any continuum source terms were then distributed into broader continuum groups that fill the energy range between peak groups.

Once the energy group structure is determined using this adaptive algorithm, the group structure was used to generate a problem-specific photon cross section library using Coupled Electron-Photon Cross Section (CEPXS) [9]. The spatially dependent neutron-induced gamma spectrum and the photon cross section library were then used in Attila ${ }^{\circledR}$ to calculate the photon transport to the detector location. RADSAT photon-transport calculations use a combination of analytical ray tracing, integral transport, and discrete ordinates $S_{N}$ methods - an approach capable of mitigating ray effects for many radiation-detection scenarios. The result of these calculations is a multi-group, discrete ordinates $S_{N}$ angular flux at all points in the model. The angular flux can then be used as a boundary condition for subsequent MCNP pulseheight tally calculations at the detector location

\subsection{Gamma-Ray Spectrometer Response}

The multi-group photon angular flux at the detector location was calculated in the photon transport calculations and was used as the source term for a "near-field” Monte Carlo simulation for pulse-height tally in the gamma-ray spectrometer. The pulse-height tally was calculated using MCNP5 within RADSAT in this work and recorded in uniform, narrow energy bins ( $1 \mathrm{keV}$ wide). A detailed explanation of the mechanics and utility of the RADSAT coupling method was provided in [3].

\subsection{Results and Discussion}

A PINS geometry was defined, consistent with field practices. That geometry is shown in Figure 2, where the mortar is filled with a common chemical weapon or the munition is replaced with a surrogate such as: graphite, water, sulfur, mustard gas (HD), FS smoke (FS), nerve agent (GB), and persistent nerve agent (VX). The mortar is interrogated using a californium neutron source and a high-resolution gamma-ray spectrometer is used to observe the induced photon spectrum within the chemical munition.

The ${ }^{252} \mathrm{Cf}$ neutron source was placed inside a polyethylene source holder with a bismuth cover. A tungsten block was placed between the source and the bismuth-collimated HPGe detector in order to provide some shielding for the collimated detector from the neutron source, in addition to the polyethylene source holder. The HPGe spectrometer was modeled after a detector used in previous PINS measurements and had a relative efficiency of $40.7 \%$. This configuration is shown in Figure 2. 
PNNL-18793

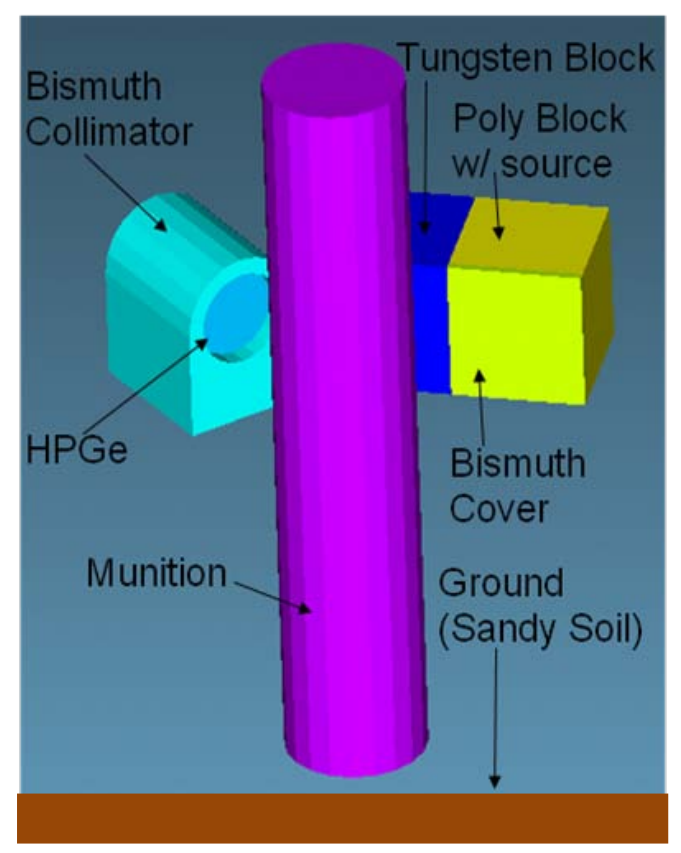

Figure 2. PINS Configuration used in RADSAT Modeling

RADSAT calculations were compared to empirical data taken by Idaho National Laboratory (INL) on actual unexploded munitions in the field and surrogate materials in PINS configurations.

\subsection{Graphite}

The graphite benchmark case is a useful first test case because of the relatively small number of neutron reactions that generate gamma rays, and the fact that all induced photons are discrete (no continuum production from carbon). This is similar to the polyethylene test case implemented in the initial testing for RADSAT coupled neutron-gamma problems [4], except in a more complex geometry. The 4-inch by 4inch by 4-inch block of graphite was modeled to replace the munition in the PINS configuration in Figure 2. It was located $10.75 \mathrm{~cm}$ from the right edge of the source and $10 \mathrm{~cm}$ from the front edge of the bismuth shield.

The experimental results provided by INL are shown below in Figure 3. The live time for the spectrum was 6000 seconds. The 4.439 and $4.497 \mathrm{MeV}$ gamma rays for carbon are observed in the data, but the 1.263 and $3.684 \mathrm{MeV}$ gamma rays are not. This may be due to the low production rates of these gamma rays or it may be due to inconsistencies in the energies tabulated in the cross section libraries versus the energies observed in the laboratory measurements. In addition to identifying carbon in the sample, the doublet for iron (7.631 and 7.645 MeV) and the hydrogen capture gamma ray (2.223 MeV) were observed. 


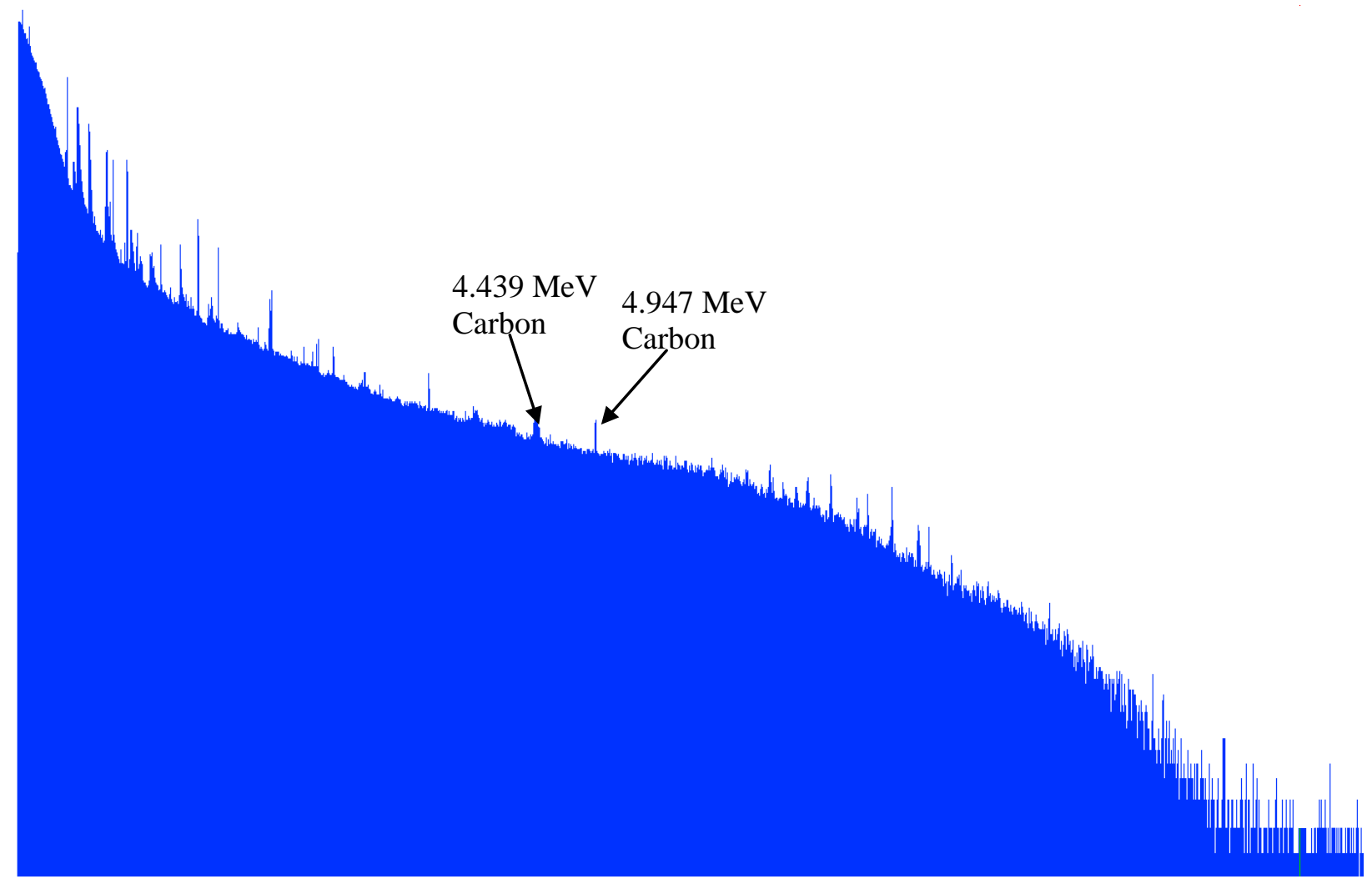

Figure 3. Empirical data from the irradiation of graphite blocks (Data provided by INL).

Due to the format of the empirical data, it was not possible to directly compare the empirical data to the MCNP and RADSAT data. A comparison of the spectra generated using MCNP and RADSAT is shown in Figure 4.

Although neither the MCNP or RADSAT spectra closely resemble the empirical data for the graphite irradiated sample, the MCNP results appear more reasonable. This is mainly because the only activation simulated in the detector response in the RADSAT model is the carbon activation spectra and the 511 $\mathrm{keV}$ gamma ray manually inserted using the RADSAT GUI. In order to obtain the full detector response obtained using MCNP, each material in the model would need to be specified, not just the material of interest. For these more complex geometries, such as PINS, the additional materials present contribute significantly to the observed spectrum thus reducing the accuracy of modeling only the material of interest in the model when comparing to empirical data or MCNP.

The neutron flux in the graphite blocks calculated using MCNP and RADSAT were a $2.85 \times 10^{-3}$ and $2.94 \times 10^{-3}$ neutrons $/ \mathrm{cm}^{2} / \mathrm{sec} /$ source particle, respectively. The gamma flux in the graphite blocks calculated using MCNP and RADSAT were a $2.52 \times 10^{-4}$ and $1.95 \times 10^{-6}$ gammas $/ \mathrm{cm}^{2} / \mathrm{sec} /$ source particle, respectively. It is not possible to directly tally the production of all gamma rays produced in MCNP but it is possible to determine the exact number of gamma rays produced in RADSAT. The large discrepancy between the number of gamma rays present in the graphite block in MCNP and the number produced in RADSAT is likely due to the lack of production of gamma rays by other isotopes in the problem besides carbon. 


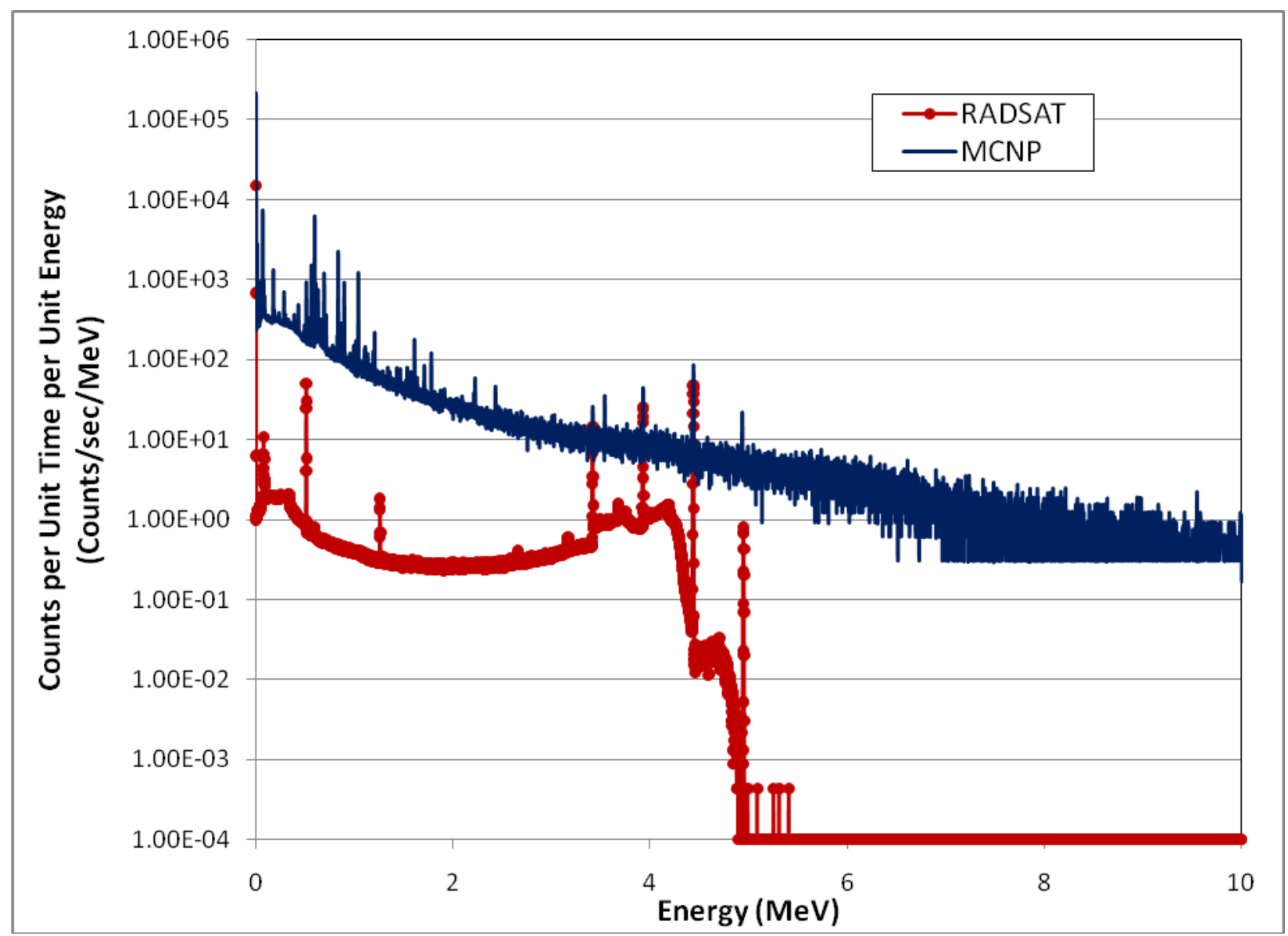

Figure 4. Comparison of MCNP and RADSAT detector response for graphite irradiated in PINS configuration.

\subsection{Water}

Two separate water benchmark cases were modeled, one using a 1-Liter water bottle and one using a 4Liter water bottle, using both MCNP and RADSAT and compared to empirical data. Each of the water bottles replaced the munition in a typical PINS configuration. Varying the amount and configuration of the water present changes the moderation of the neutrons within the volume of water, thus changing the photon spectrum produced.

Water represents a material of intermediate complexity in terms of coupled neutron-photon problems in that $\mathrm{H}_{2} \mathrm{O}$ has ten gamma-producing reactions in the neutron energy range included in the Kynea3 neutron energy group structure ( $1 \mathrm{e}^{-5} \mathrm{eV}$ to $19.64 \mathrm{MeV}$ ), but none of those reactions have strong resonances. There are a total of 119 discrete gamma rays and one continuum produced by neutron activation in ${ }^{16} \mathrm{O}$.

The 1-Liter water bottle was modeled as a 3.64-inch diameter bottle in place of a chemical munition. The center of the bottle is $15.75 \mathrm{~cm}$ from the source hole and $16 \mathrm{~cm}$ from the bismuth shield. The 4-Liter water bottle was modeled as two Nalgene bottles, $242 \mathrm{~mm}$ by $84 \mathrm{~mm}$ by $152 \mathrm{~mm}$, each. The Nalgene bottles were placed $9.4 \mathrm{~cm}$ from the right edge of the source and $8 \mathrm{~cm}$ from the front of the bismuth shield. In both of the simulations, the bottles were neglected from the model since the thickness of the plastic should have negligible impact on the induced spectra. 
The experimental results provided by INL for the 1-Liter and 4-Liter water bottle irradiations are shown in Figure 5 and Figure 6, respectively. Both the 1-Liter and 4-Liter water bottle irradiation data have live times of 1000 seconds. The most prominent peak observed is the $2.223 \mathrm{MeV}$ produced by neutron capture in hydrogen. The hydrogen capture gamma ray and the Compton edge are significantly more pronounced for the irradiation of the 4-Liter water bottle than the 1-Liter water bottle. All other aspects of the spectra are essentially the same.

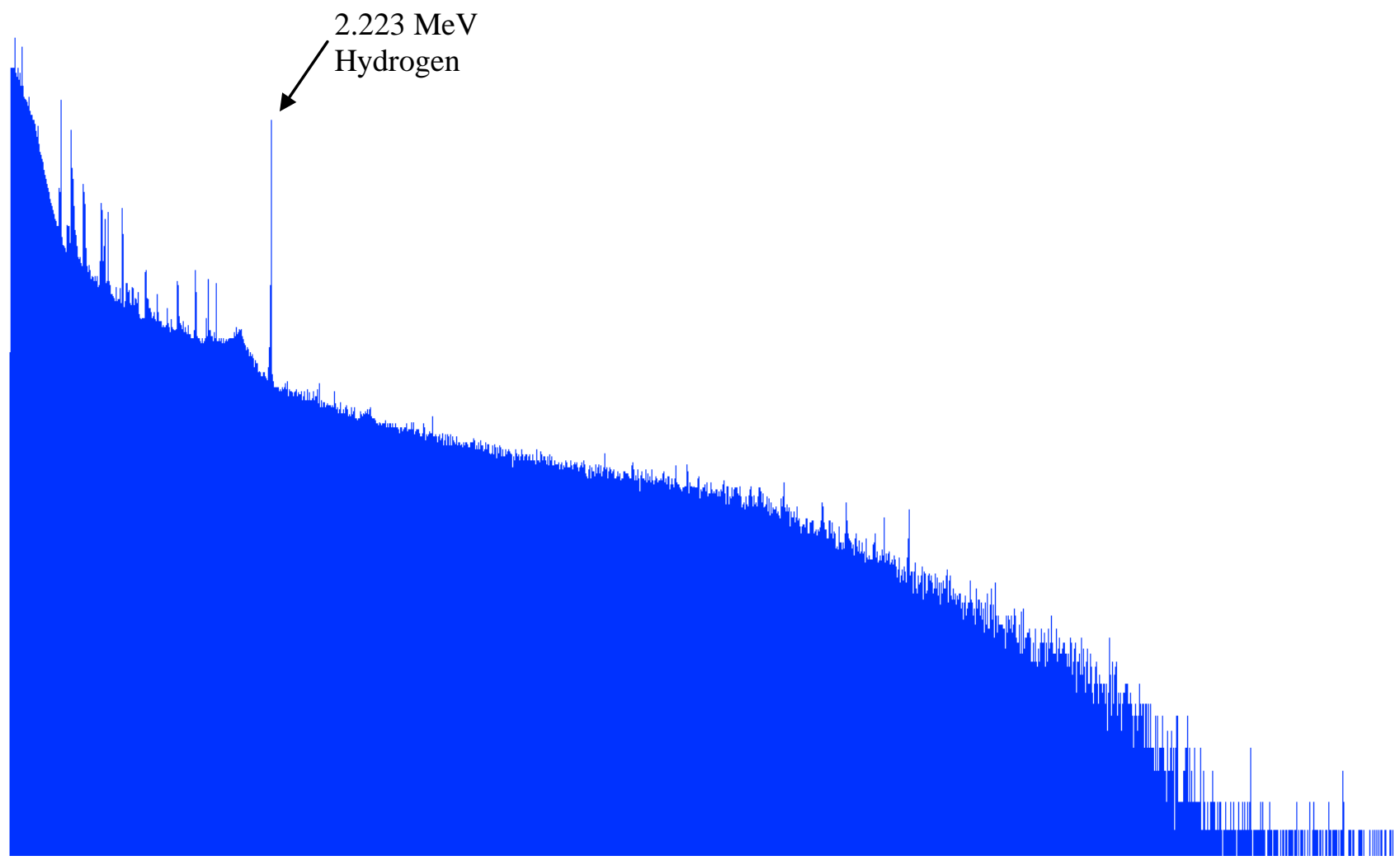

Figure 5. Empirical data from the irradiation of a 1-Liter water bottle (Data provided by INL). 


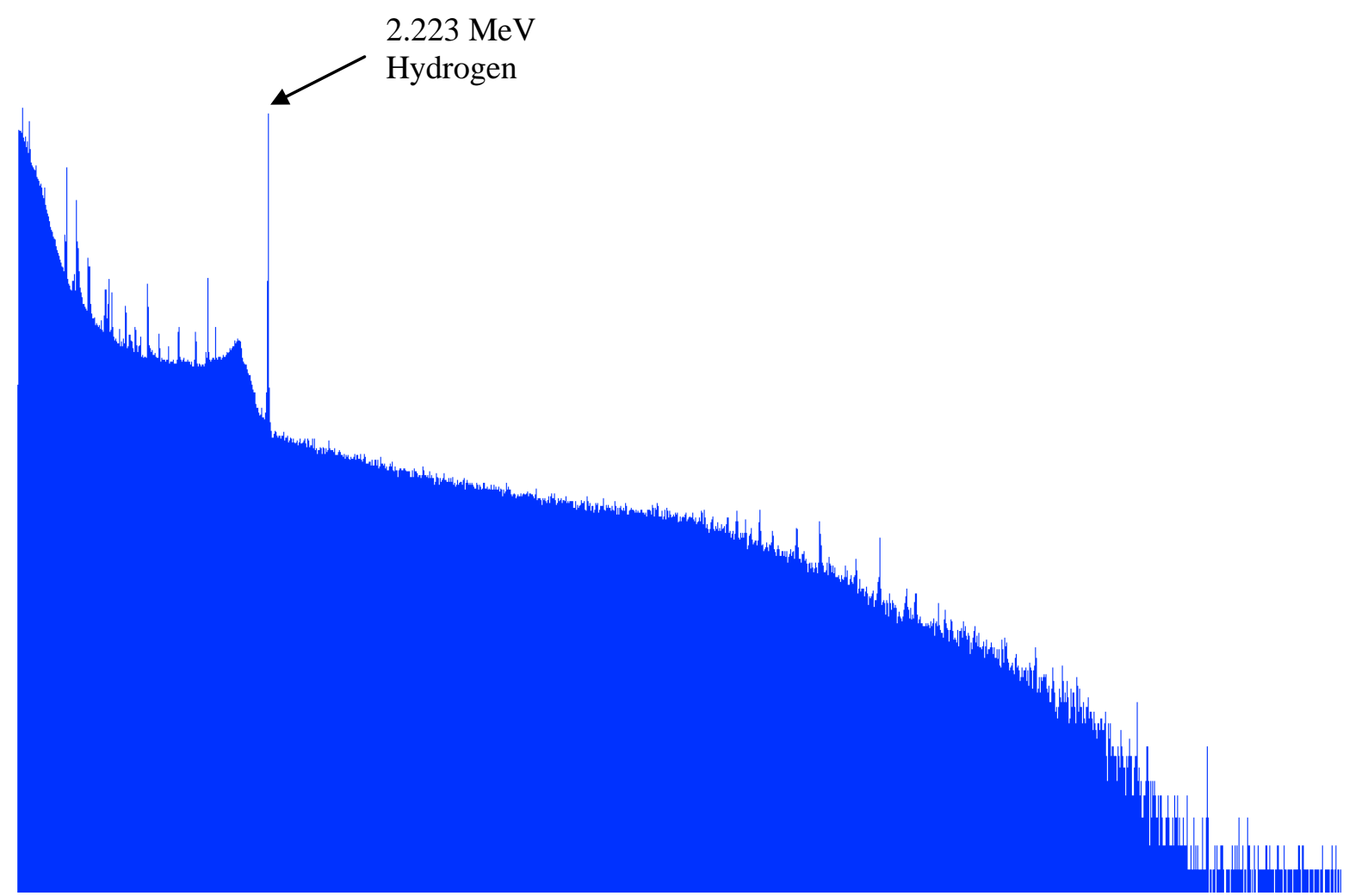

Figure 6. Empirical data from the irradiation of a 4-Liter water bottle (Data provided by INL).

A comparison of the gamma ray spectra generated using MCNP and RADSAT are shown in Figure 7 and Figure 8, respectively. Similar issues due to the RADSAT modeling assumptions are present with the irradiated water spectra in the PINS configuration. The neutron flux in both the 1-Liter and 4-Liter benchmark cases were comparable for the MCNP and RADSAT cases but there were significantly more gamma rays present in the MCNP in the water and the detector response than were created using RADSAT. From the initial benchmarking cases done with MCNP [4][5], it is believed that this discrepancy is due to the addition of the complex geometry and not including the gamma production due to these components in the problem, not due to deficiencies in the cross section preparations. 


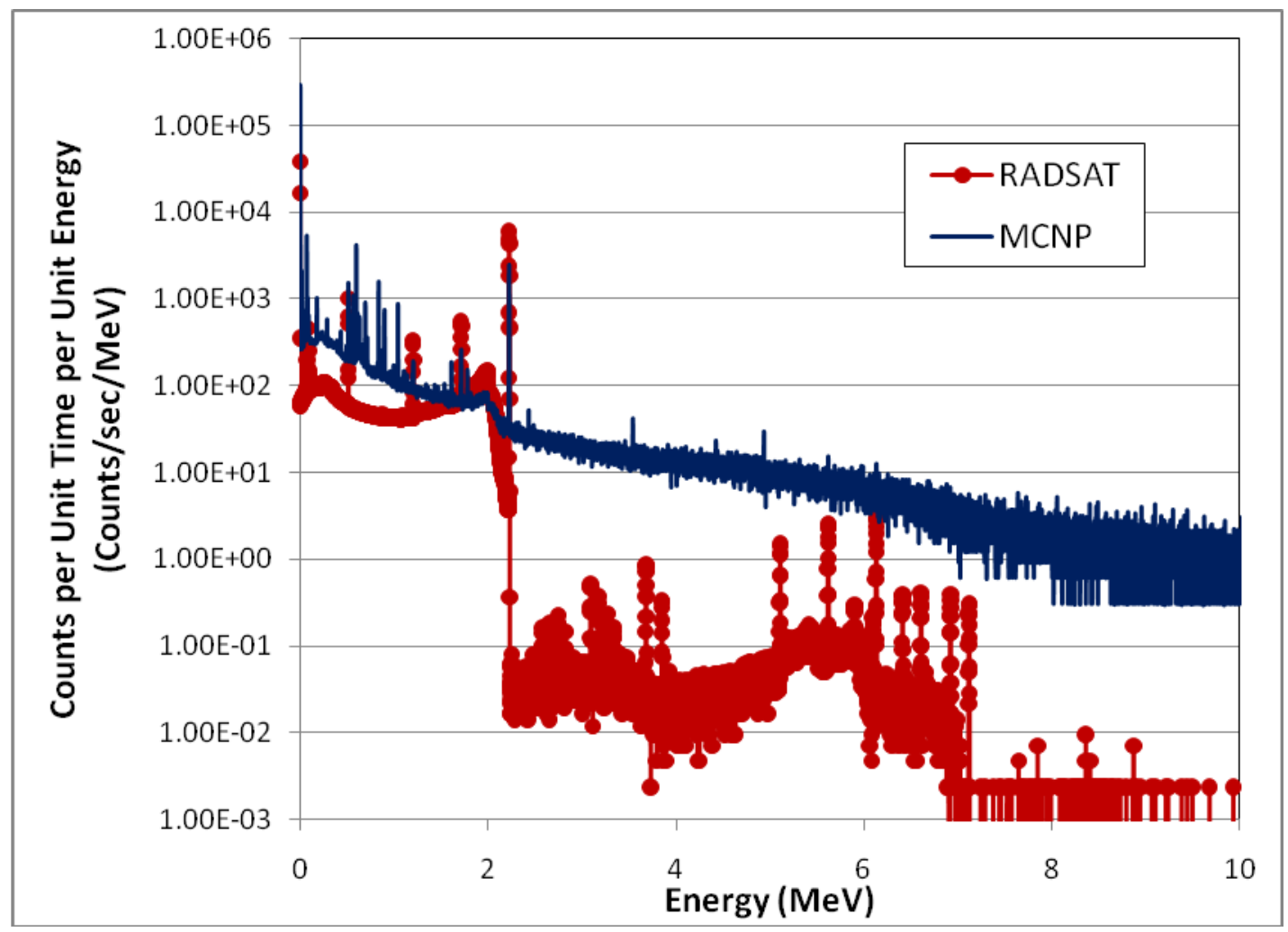

Figure 7. Comparison of MCNP and RADSAT detector response for 1-Liter water bottle irradiated in PINS configuration.

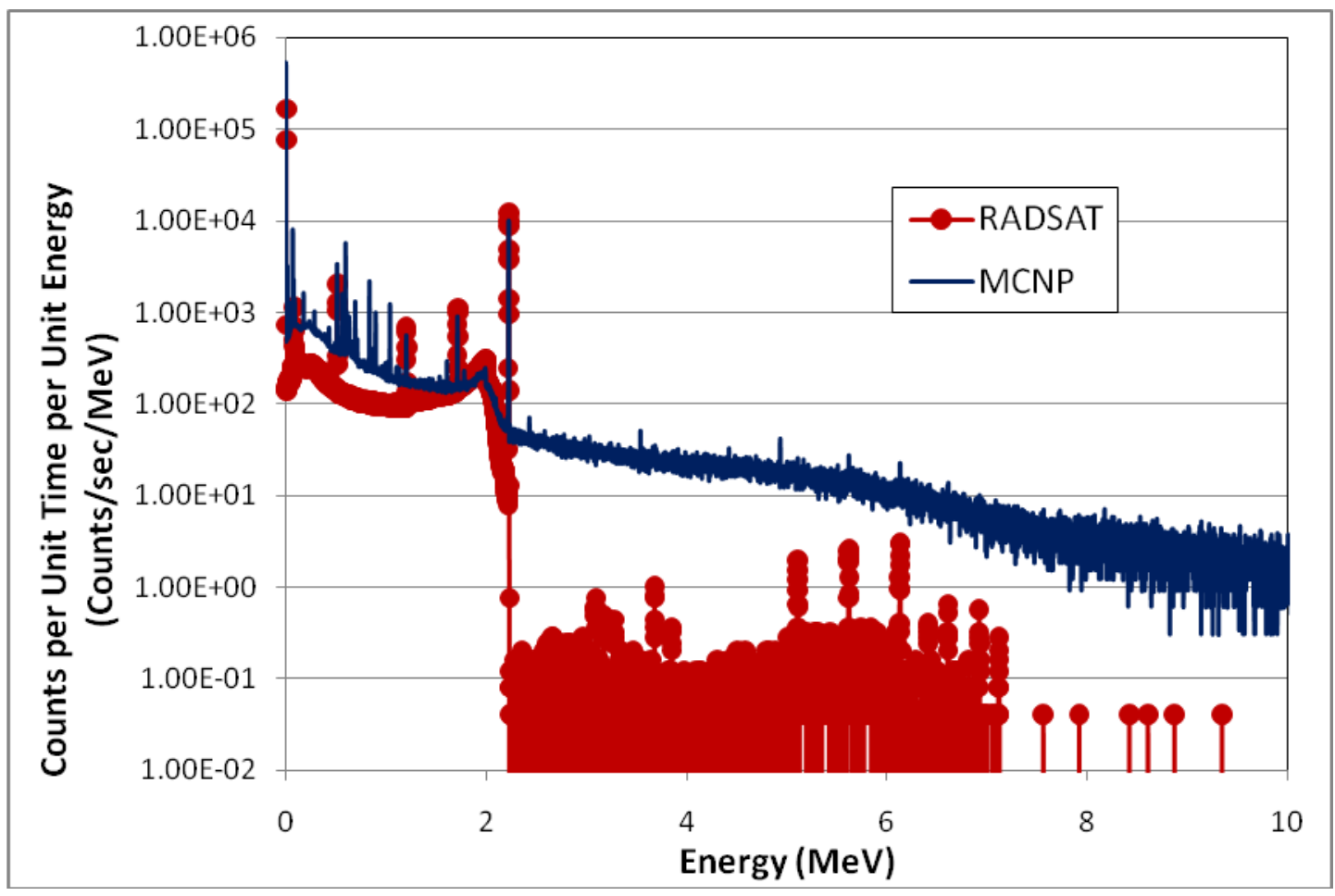

Figure 8. Comparison of MCNP and RADSAT detector response for 4-Liter water bottle irradiated in PINS configuration. 
Originally, both the MCNP and RADSAT calculations were completed using ENDF/B-VII cross sections to simulate the gamma ray production in the water. The MCNP energy bins are set to be uniformly spaced at $1.1 \mathrm{keV}$ bin widths. At this energy resolution it was not obvious without close examination that the hydrogen peak, historically recorded at $2.2233 \mathrm{MeV}$ [16], was moved slightly to $2.224631 \mathrm{MeV}$ [15]. When the RADSAT calculation was completed with an energy resolution of $0.25 \mathrm{keV}$ bin width, the energy discrepancy became apparent. Upon further investigation, it was discovered that the ENDF/B-VII cross section library has changed the reported neutron capture gamma ray energy for hydrogen from 2.2233 MeV to $2.224631 \mathrm{MeV}$. Since the energy of the hydrogen peak is paramount to the PINS application, it was decided that all RADSAT calculations requiring hydrogen shall use ENDF/B-VI cross sections for hydrogen. The MCNP calculations were completed using ENDF/B-VII cross sections for hydrogen however for comparison.

\subsection{Sulfur}

Sulfur represents an important material in a PGNAA application since it is a common constituent of chemical munitions. There are four naturally occurring isotopes of sulfur (natural isotopic ratios: ${ }^{32} \mathrm{~S}-$ $94.93 \%,{ }^{33} \mathrm{~S}-0.76 \%,{ }^{34} \mathrm{~S}-4.29 \%$, and ${ }^{36} \mathrm{~S}-0.02 \%$ ), all are contained in the ENDF/B-VII cross section library. Sulfur produces 28 continua and 57 discrete gamma rays.

It should be noted that the 2.379 and $5.42 \mathrm{MeV}$ sulfur lines are not present in the original ENDF/B-VII cross section data but are present in the ENDF/B-VI cross section data. The RADSAT-NG cross section library was derived from the ENDF/B-VII cross section library. Since the 2.379 and $5.42 \mathrm{MeV}$ sulfur lines are important Portable Isotopic Neutron Spectroscopy (PINS) [11], the cross section for the production of the $2.379 \mathrm{MeV}$ and $5.42 \mathrm{MeV}$ lines were taken from the ENDF/B-VI cross section library for ${ }^{32} \mathrm{~S}$ and added to the RADSAT-NG ${ }^{32} \mathrm{~S}$ cross section library.

It was determined that the addition of these two cross sections to ENDF/B-VII was more accurate for modeling PINS applications than applying the ENDF/B-VI cross sections to this problem. The ENDF/BVI cross sections was missing the $2.230 \mathrm{MeV}$ gamma ray and the continuum produced from this data did not correspond with the general shape of the empirical data. The MCNP calculations apply the un-alter ENDF/B-VII cross sections for comparison.

The sulfur was modeled in the same configuration as the experimental setup, with a 3-inch diameter bottle with the sulfur filling the bottle to a height of 2.2 inches. The bottle was neglected from the model since the thickness of the plastic should have negligible impact on the induced spectrum. The sulfur was place $15.75 \mathrm{~cm}$ from the source hole and $16 \mathrm{~cm}$ from the bismuth shield in place of the munition in the standard PINS configuration shown in Figure 2. The experimental results provided by INL for the flaked sulfur irradiation is shown in Figure 9 with a live time of 6000 seconds. The three major characteristic gammarays for sulfur (2.23 MeV, $2.379 \mathrm{MeV}$ and $5.42 \mathrm{MeV}$ ) are clearly observable in this spectrum. 


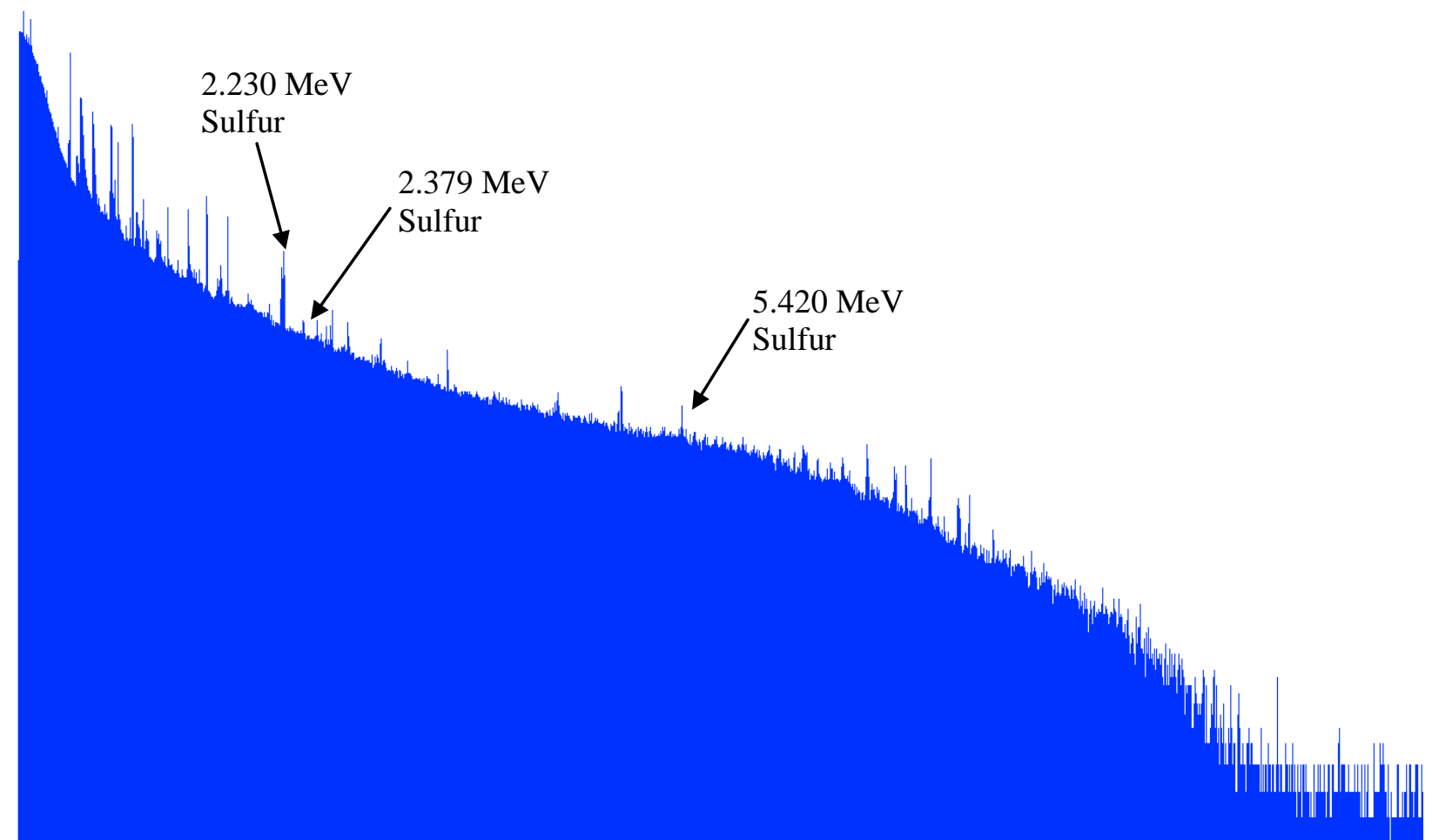

Figure 9. Empirical data from the irradiation of flaked sulfur (Data provided by INL).

A comparison of the spectra generated using MCNP and RADSAT is shown in Figure 10. Similar to the issues present in the water and graphite irradiation, the spectrum for the irradiation of sulfur is not consistent for the RADSAT model, although it begins to improve. This is due to the complexity of the gamma spectrum produced by the isotopes of sulfur. The complexity of these spectra begins to hide some of the insufficiencies in the RADSAT simulation from the missing data from the aspects of the problem that are not being adequately modeled. 


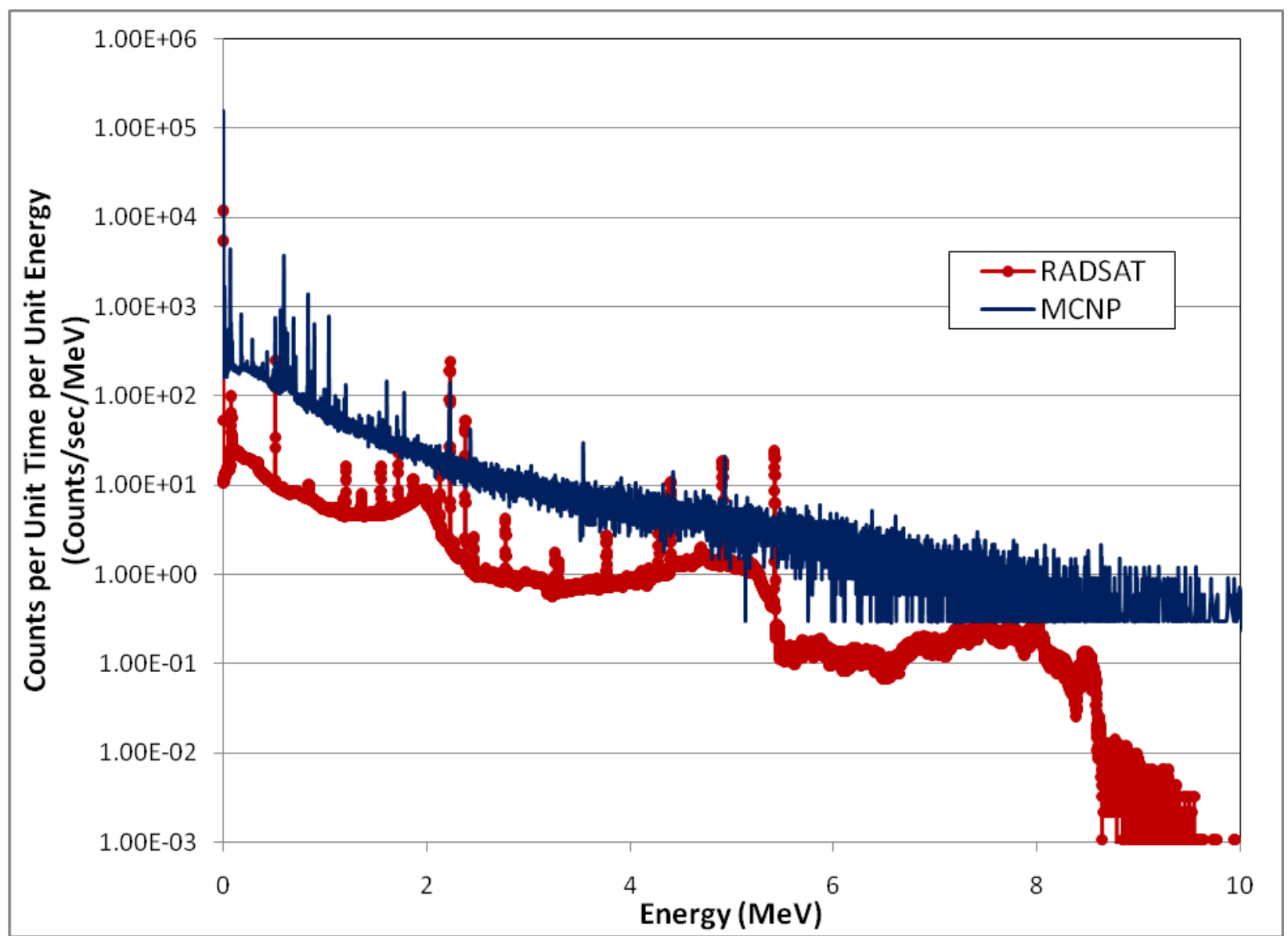

Figure 10. Comparison of MCNP and RADSAT detector response for sulfur irradiated in PINS configuration.

\subsection{Mustard Gas (HD)}

In this benchmark case, a 4.2-inch mortar was simulated. The modeled HD assumes a nominal composition of $30.2 \%$ carbon, $44.7 \%$ chlorine, $5.0 \%$ hydrogen, and $20.1 \%$ sulfur [11] and has a density of 1.27 grams $/ \mathrm{cm}^{3}$ [12], but it should be noted that in reality there are ranges for both, particularly the composition. The mortar casing was modeled as carbon steel, which is composed of $0.06 \%$ carbon, $0.35 \%$ manganese, and 99.59\% iron and has a density of $7.872 \mathrm{grams} / \mathrm{cm}^{3}$ in both the MCNP and RADSAT models.

The experimental results provided by INL for the HD irradiation is shown in Figure 11 with a live time of 6000 seconds. The major characteristic gamma-rays for sulfur (2.23 MeV), hydrogen (2.223 MeV), chlorine (1.950 and 1.959 MeV), and iron (7.631 and 7.645 MeV) are clearly observable in this spectrum. 


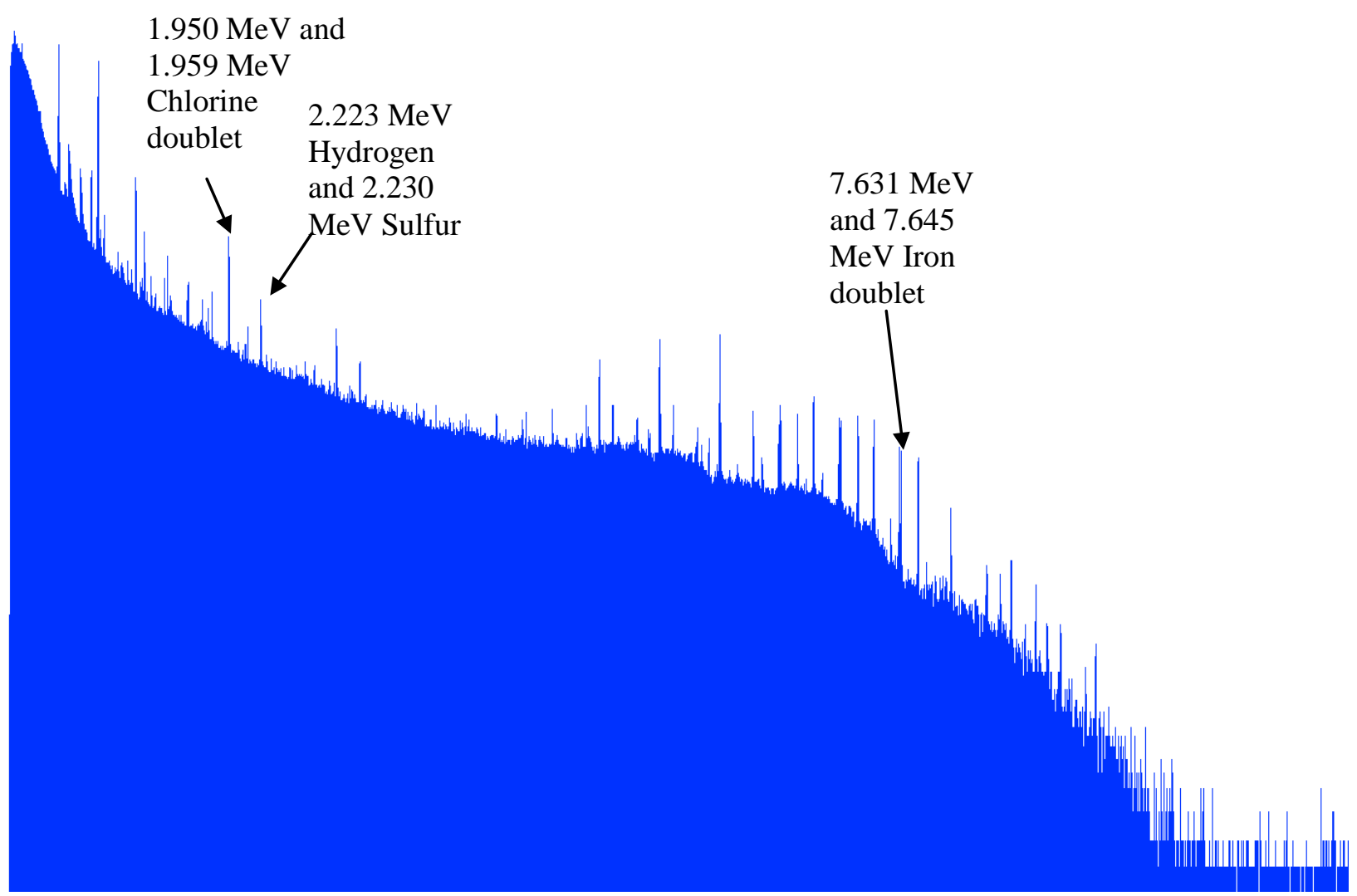

Figure 11. Empirical data from the irradiation of HD (Data provided by INL).

The comparison of the MCNP and RADSAT results for the HD irradiation is shown in Figure 12. The MCNP models employs unmodified ENDF/B-VII cross section libraries while the RADSAT results incorporate the modified RADSAT-NG ENDF/B-VII cross section library. As described in previous sections, the modified ENDF/B-VII cross section library was augmented to include the $2.379 \mathrm{MeV}$ and $5.42 \mathrm{MeV}$ sulfur gamma rays. It also uses the ENDF/B-VI hydrogen cross section in place of the ENDF/B-VII cross section to maintain the proper energy for the hydrogen capture gamma ray.

A comparison of the peak count rates for the regions of interest peak ratios are provided in Table 1. The simulated count peak rates were on average an order of magnitude lower than the empirical data, but this may have been caused by a processing error. To complete the comparison of simulated and measured PINS assays, we compare the $\mathrm{Cl} / \mathrm{S}$ peak ratio, which is a key indicator for chemical weapon composition. PINS experience indicates that for HD, this ratio should be between 10 and 20 [9]. A comparison of RADSAT and empirical peak ratios are shown in Table 2. Both the MCNP and RADSAT calculation fall outside of the range of acceptable $\mathrm{Cl} / \mathrm{S}$ peak ratio for $\mathrm{HD}$. This may be due to the bin structure used in the detector model or in the cross section library implemented.

Table 1. Peak count rates for regions of interest from the irradiation of HD.

\begin{tabular}{|c|c|c|c|c|c|c|}
\hline & $1.95 \mathrm{MeV}$ & $1.959 \mathrm{MeV}$ & $2.223 \mathrm{MeV}$ & $2.23 \mathrm{MeV}$ & $7.631 \mathrm{MeV}$ & $7.645 \mathrm{MeV}$ \\
\hline Empirical & 4688 & 3287 & 1589 & 301 & 300 & 284 \\
\hline MCNP & 513 & 352 & 207 & 69.6 & 49.5 & 43.8 \\
\hline RADSAT & 901 & 614 & 171 & 74.9 & 42.4 & 38.5 \\
\hline
\end{tabular}


Table 2. Comparison of peak ratio for irradiation of $\mathrm{HD}$.

\begin{tabular}{|c|c|}
\hline & Ratio Cl (1959) / S (2230) \\
\hline Empirical & 10.9 \\
\hline MCNP & 5.06 \\
\hline RADSAT & 8.19 \\
\hline
\end{tabular}

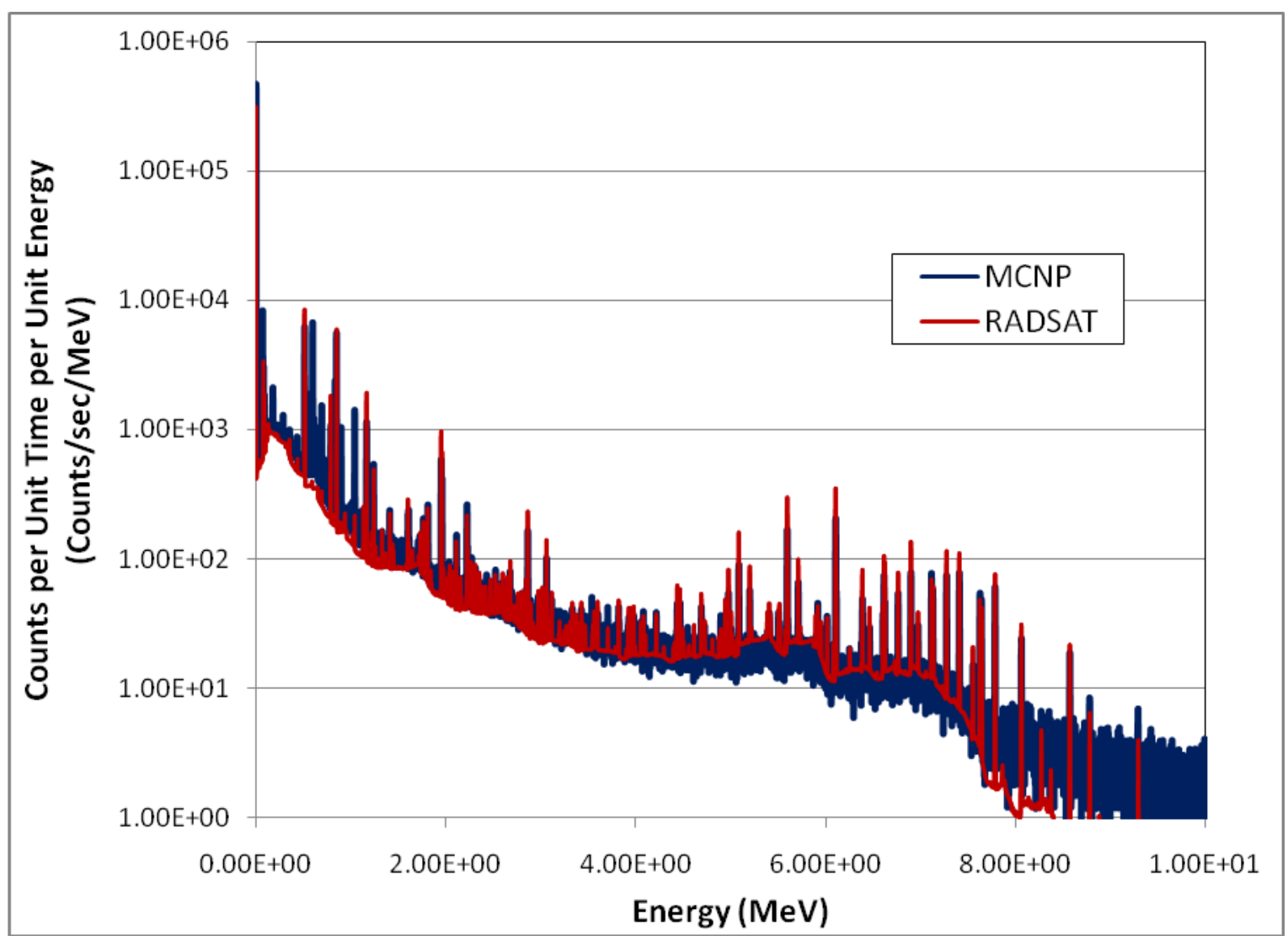

Figure 12. Comparison of MCNP and RADSAT detector response for HD irradiated in PINS configuration.

Unlike with the simple surrogate models, the RADSAT method of only simulating the gamma ray production in the article of interest appears to be adequate for complex interrogation articles and geometries since they are the main contributor to the gamma ray spectrum. Since the MCNP calculation was completed using the ENDF/B-VII cross section library, while the RADSAT calculation was completed using a modified ENDF/B-VII cross section library. The peak height and placement were comparable for the peaks of interest for PINS as shown in Figures 13 thru 16 (1.95 and $1.959 \mathrm{MeV}$ chlorine doublet, 2.223 MeV hydrogen, 2.23 and 5.421 MeV sulfur, and 7.631 and 7.645 MeV iron doublet). As previously indicated the ENDF/B-VI hydrogen cross section was used for the RADSAT modeling which accounts for the slight misalignment of the two hydrogen peaks as shown in Figure 14. Additionally, the $5.42 \mathrm{MeV}$ peak is present in the RADSAT spectrum but not in the MCNP spectrum as shown in Figure 15. The transition from the gamma ray energy bins used in the deterministic transport calculation to the Monte Carlo calculation in RADSAT caused slight shifts in the peak locations and bin broadening. This phenomenon can be corrected by applying a finer energy group structure to the detector response calculation in MCNP, but this was not rectified in these calculations. 


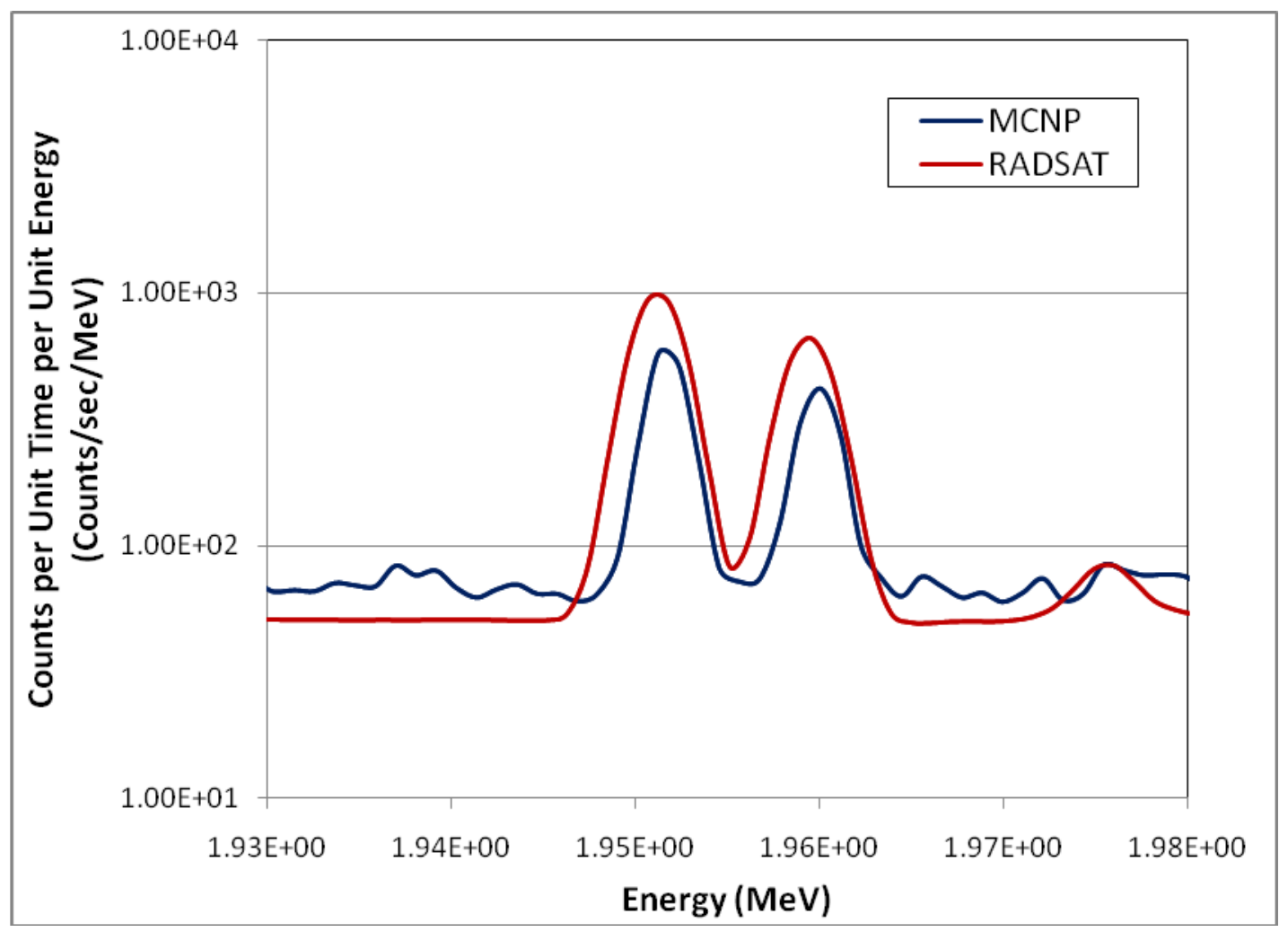

Figure 13. Comparison of 1.95 and 1.959 $\mathrm{MeV}$ chlorine doublet in MCNP and RADSAT detector response for HD irradiated in PINS configuration.

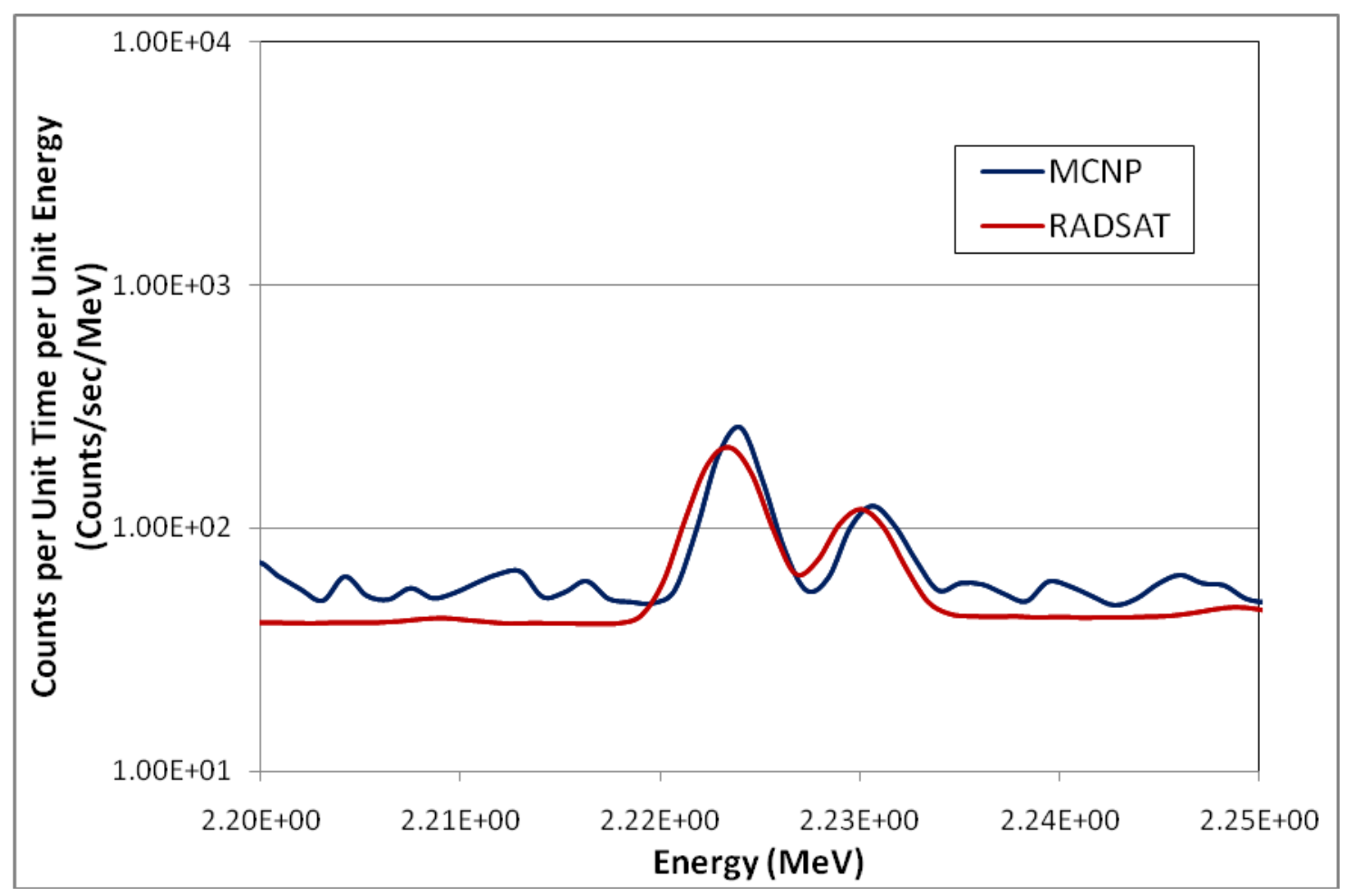

Figure 14. Comparison of 2.223 MeV hydrogen and 2.23 MeV sulfur peaks in MCNP and RADSAT detector response for HD irradiated in PINS configuration. 


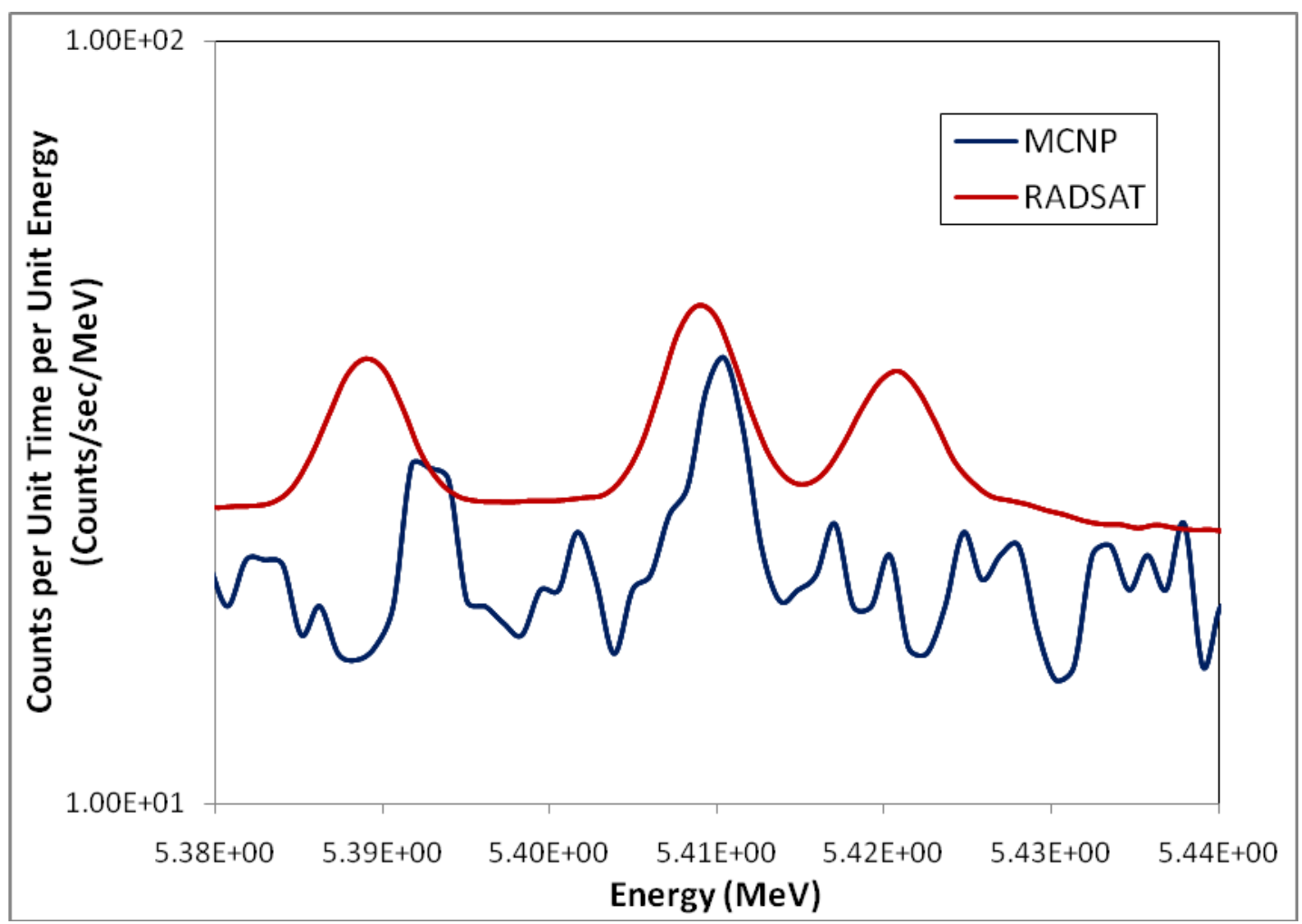

Figure 15. Comparison of 5.421 MeV sulfur peak in MCNP and RADSAT detector response for HD irradiated in PINS configuration.

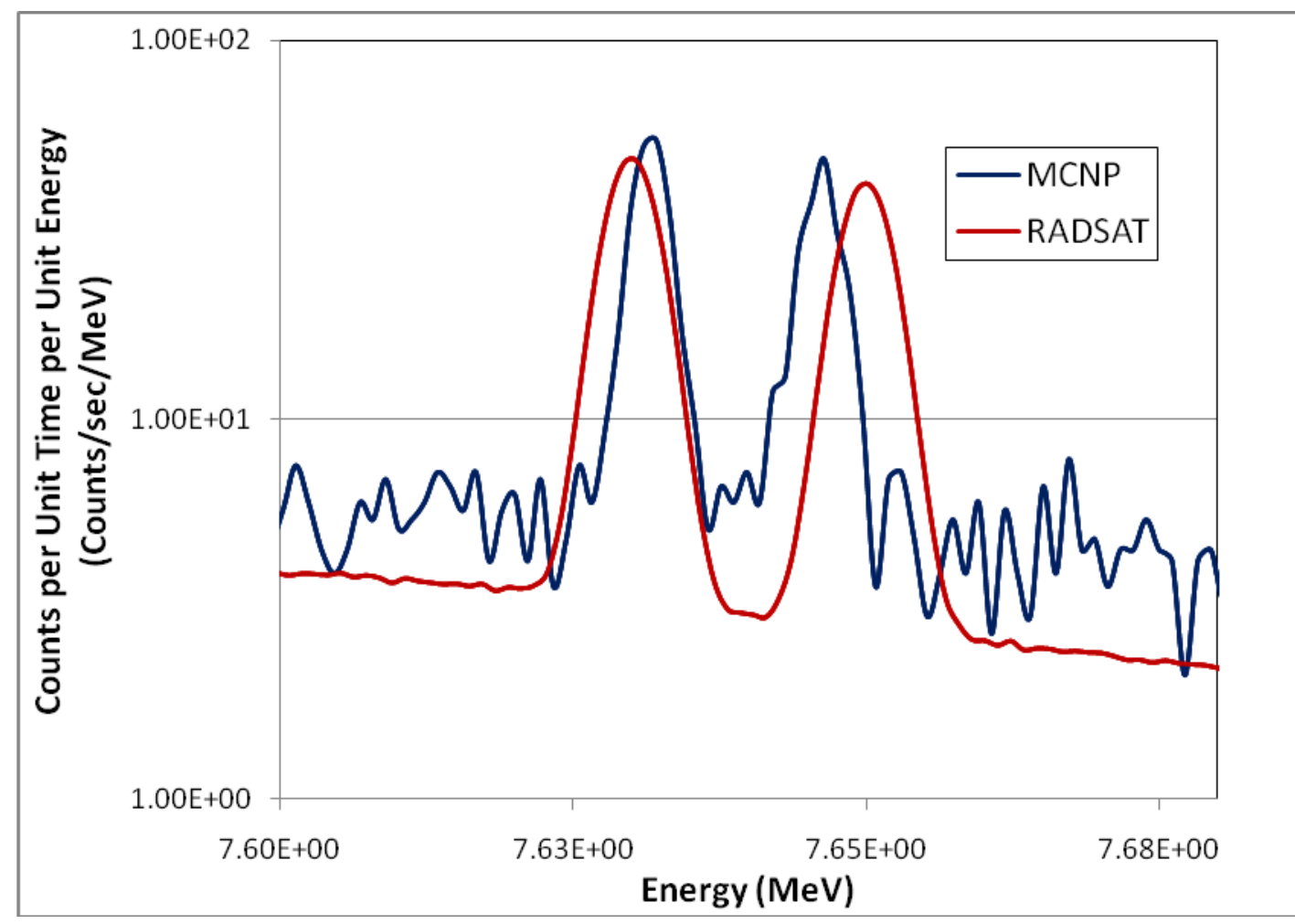

Figure 16. Comparison of 7.631 MeV and 7.645 MeV iron doublet in MCNP and RADSAT detector response for HD irradiated in PINS configuration. 


\subsection{FS Smoke (FS)}

Another important chemical weapon material for PINS applications is FS. FS was simulated in a Livens projector shell in the typical PINS configuration. The nominal FS composition was taken to be $13.7 \%$ chlorine, $0.4 \%$ hydrogen, $51.5 \%$ oxygen, and $34.4 \%$ sulfur [11] and has a density of 1.9 grams $/ \mathrm{cm}^{3}$ [13]. The experimental results provided by INL for the FS irradiation is shown in Figure 17. The major characteristic gamma-rays for sulfur (2.23 MeV), chlorine (1.950 and 1.959 MeV), and iron (7.631 and $7.645 \mathrm{MeV}$ ) are clearly observable in this spectrum.

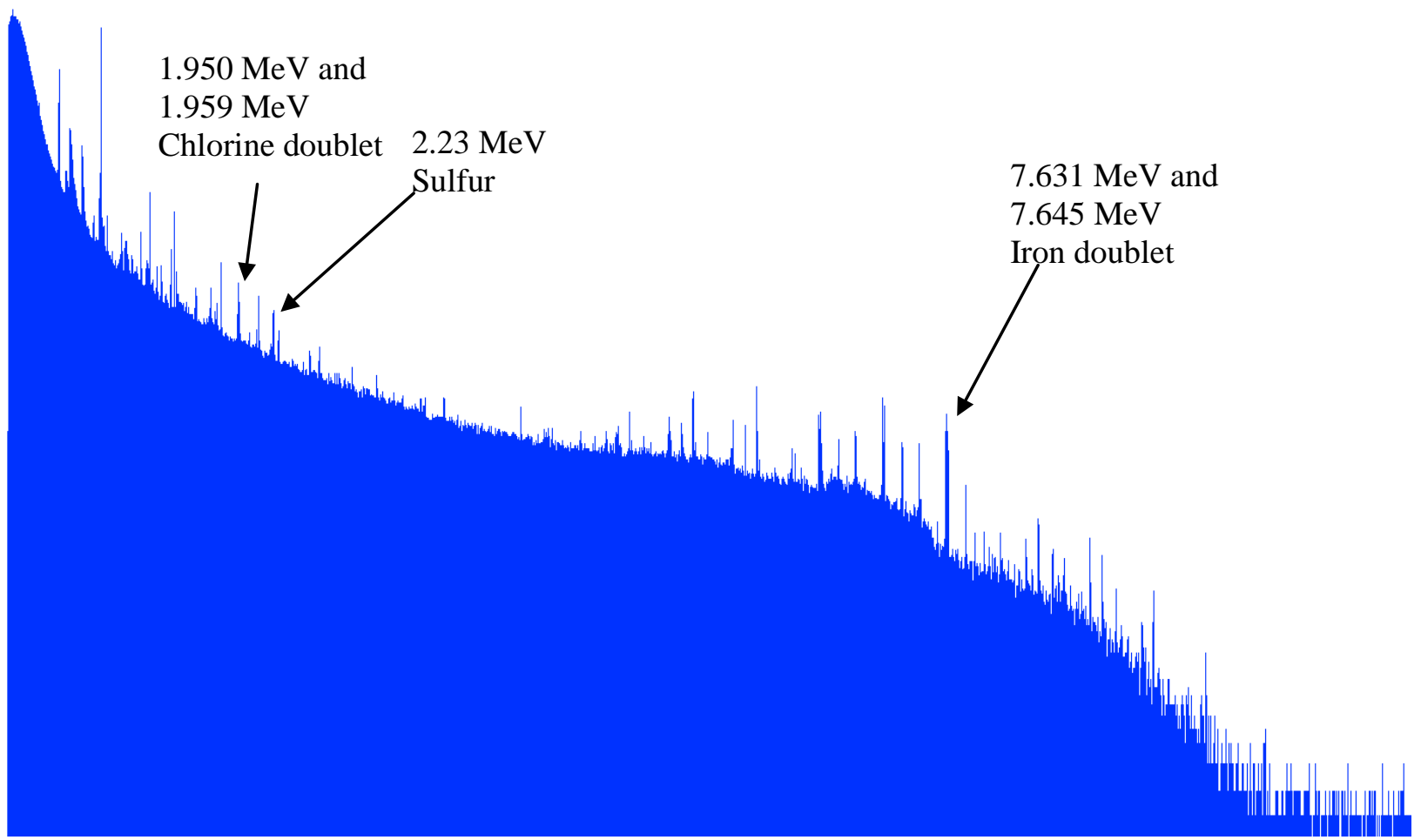

Figure 17. Empirical data from the irradiation of FS (Data provided by INL).

The comparison of the MCNP and RADSAT calculations is shown in Figure 18. Similar to the HD case, the MCNP calculation was completed using the ENDF/B-VII cross section library, while the RADSAT calculation was completed using a modified ENDF/B-VII cross section library. The energy ranges of interest for FS are shown in more detail in Figures 19 thru 21 (1.95 and 1.959 chlorine doublet, 2.23 sulfur, and 7.631 and $7.645 \mathrm{MeV}$ iron doublet). In Figure 20, a small hydrogen peak is observed in the MCNP spectra and not in the RADSAT spectra. There is no hydrogen present in the munition, but there is hydrogen in the air and the polyethylene which does not have gamma production in RADSAT. In order to obtain this gamma peak in the RADSAT spectra, it would be necessary to generate the gammas produced in the remainder of the geometries present in the problem, not just the item of interest.

A comparison of the peak count rates for the regions of interest peak ratios for FS are provided in Table 3. The PINS documentation indicates the $\mathrm{Cl} / \mathrm{S}$ ratio for FS is typically less than or equal to 2 . A comparison between RADSAT and empirical Cl/S ratios is shown in Table 4. Both the MCNP and RADSAT calculation fall outside of the range of acceptable $\mathrm{Cl} / \mathrm{S}$ peak ratio for $\mathrm{HD}$. This may be due to the bin structure used in the detector model or in the cross section library implemented. MCNP produces a $\mathrm{Cl} / \mathrm{S}$ 
ratio that was outside the acceptable range for FS. This is due to the lower chlorine peaks than calculated for RADSAT although both codes use ENDF/B-VII cross sections for chlorine.

Table 3. Peak count rates for regions of interest from the irradiation of FS.

\begin{tabular}{|c|c|c|c|c|c|}
\hline & $1.95 \mathrm{MeV}$ & $1.959 \mathrm{MeV}$ & $2.23 \mathrm{MeV}$ & $7.631 \mathrm{MeV}$ & $7.645 \mathrm{MeV}$ \\
\hline Empirical & 1800 & 1026 & 1090 & 415 & 316 \\
\hline MCNP & 258 & 194 & 537 & 30.1 & 26.4 \\
\hline RADSAT & 535 & 365 & 597 & 35.3 & 30.5 \\
\hline
\end{tabular}

Table 4. Comparison of peak ratio for irradiation of FS.

\begin{tabular}{|c|c|}
\hline & Ratio Cl (1959) / S (2230) \\
\hline Empirical & 1.06 \\
\hline MCNP & 2.78 \\
\hline RADSAT & 1.63 \\
\hline
\end{tabular}

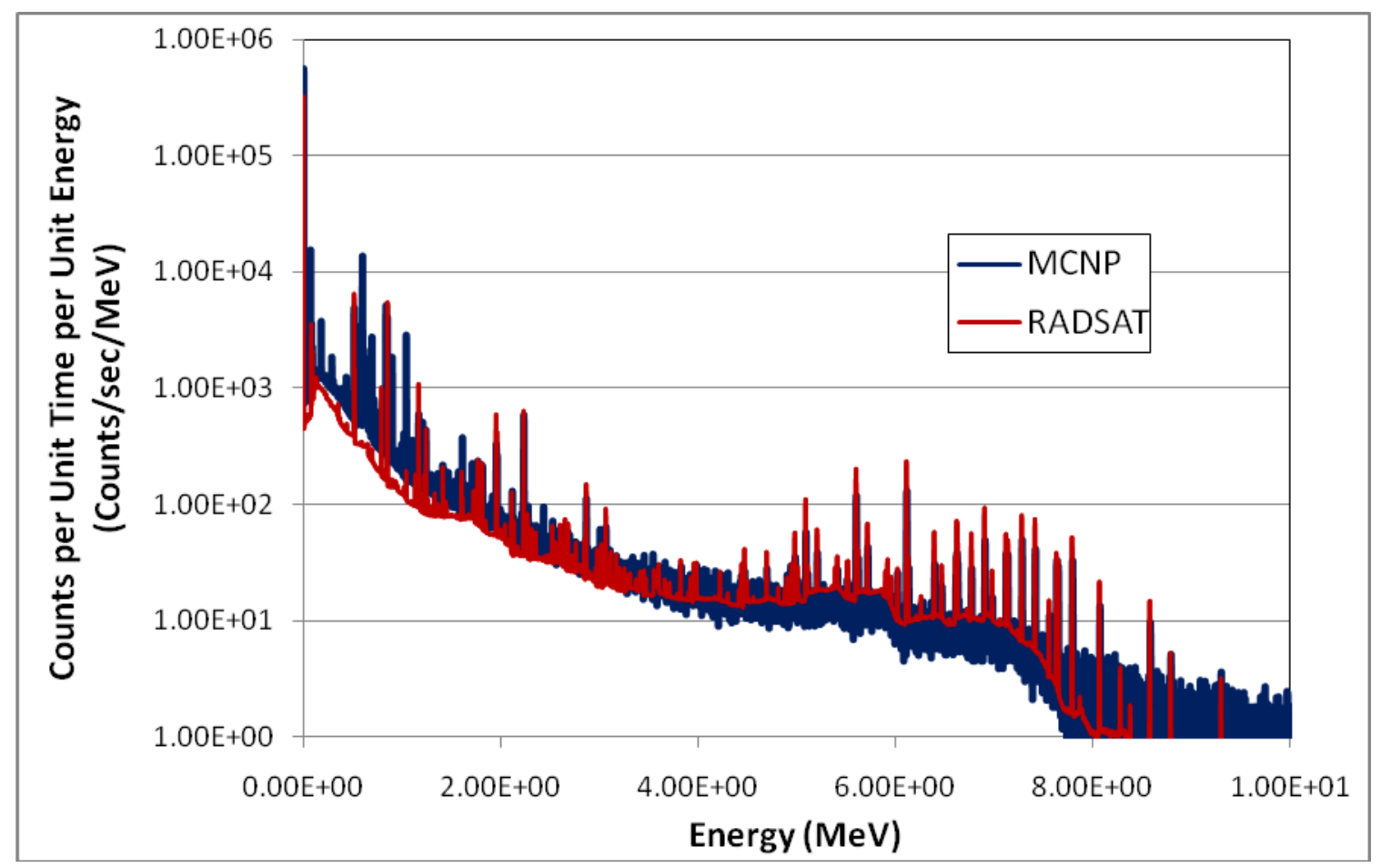

Figure 18. Comparison of MCNP and RADSAT detector response for FS irradiated in PINS configuration. 


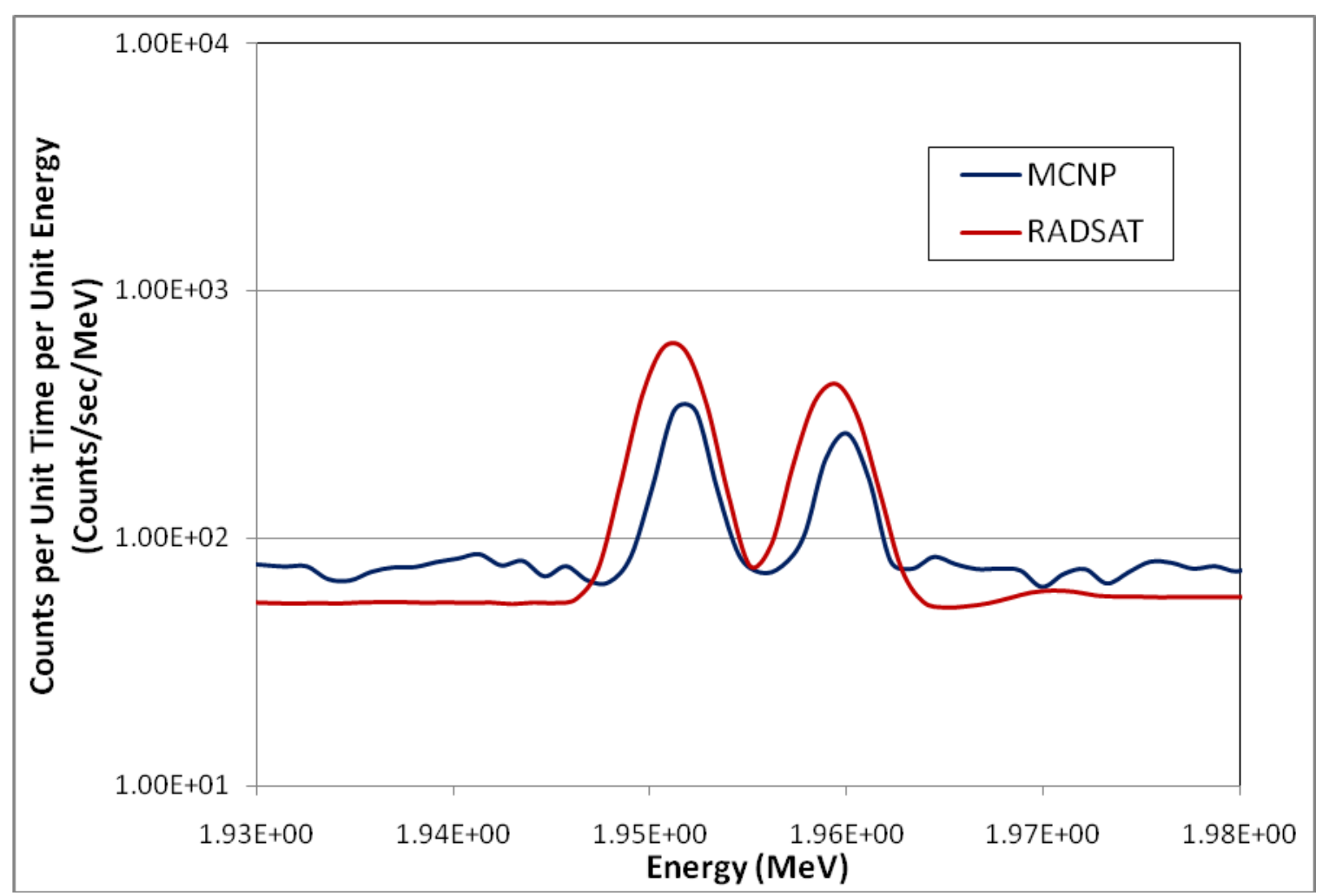

Figure 19. Comparison of 1.95 and 1.959 MeV chlorine doublet in MCNP and RADSAT detector response for FS irradiated in PINS configuration.

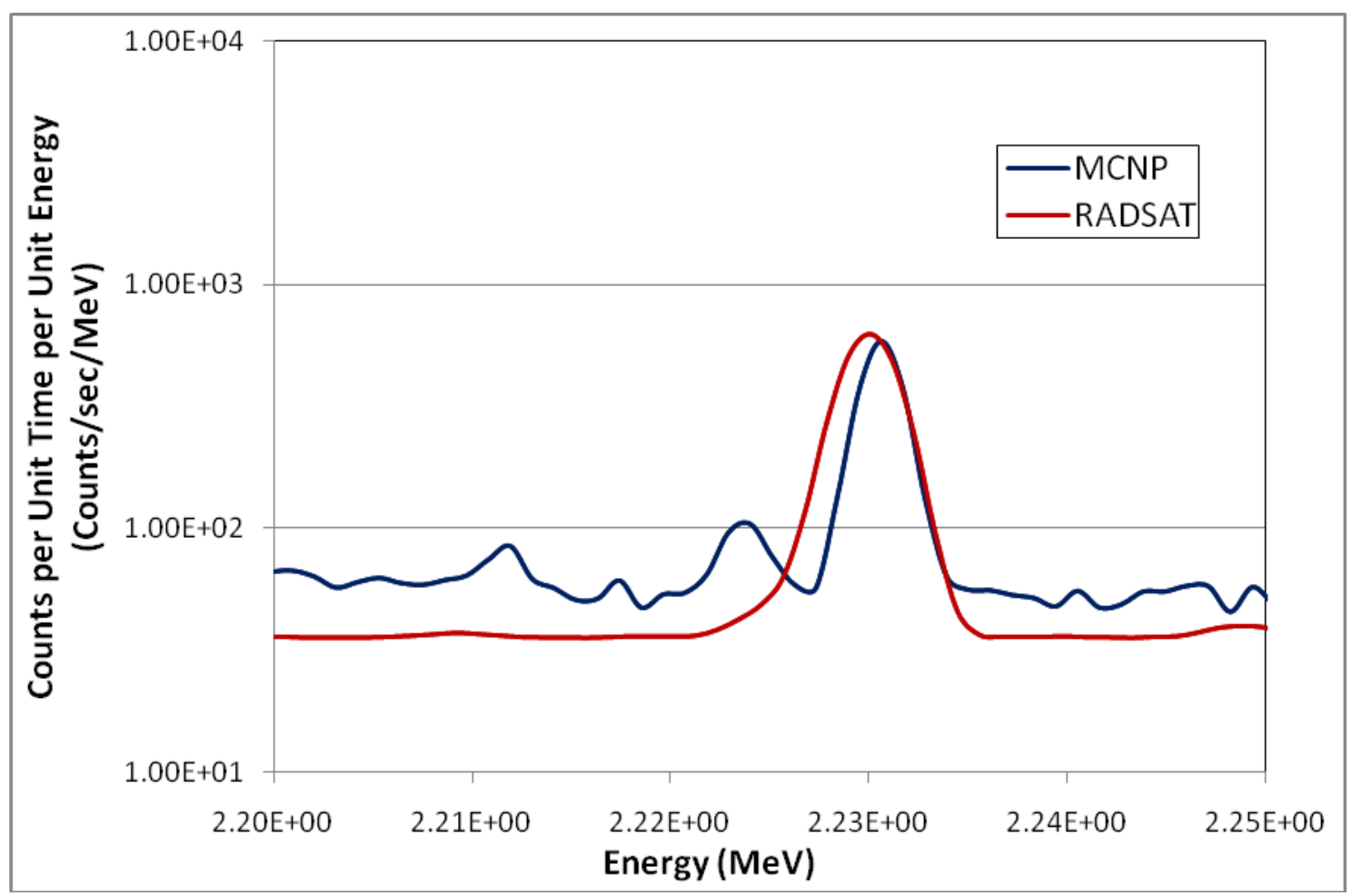

Figure 20. Comparison of 2.223 MeV hydrogen and 2.23 MeV sulfur peaks in MCNP and RADSAT detector response for FS irradiated in PINS configuration. 


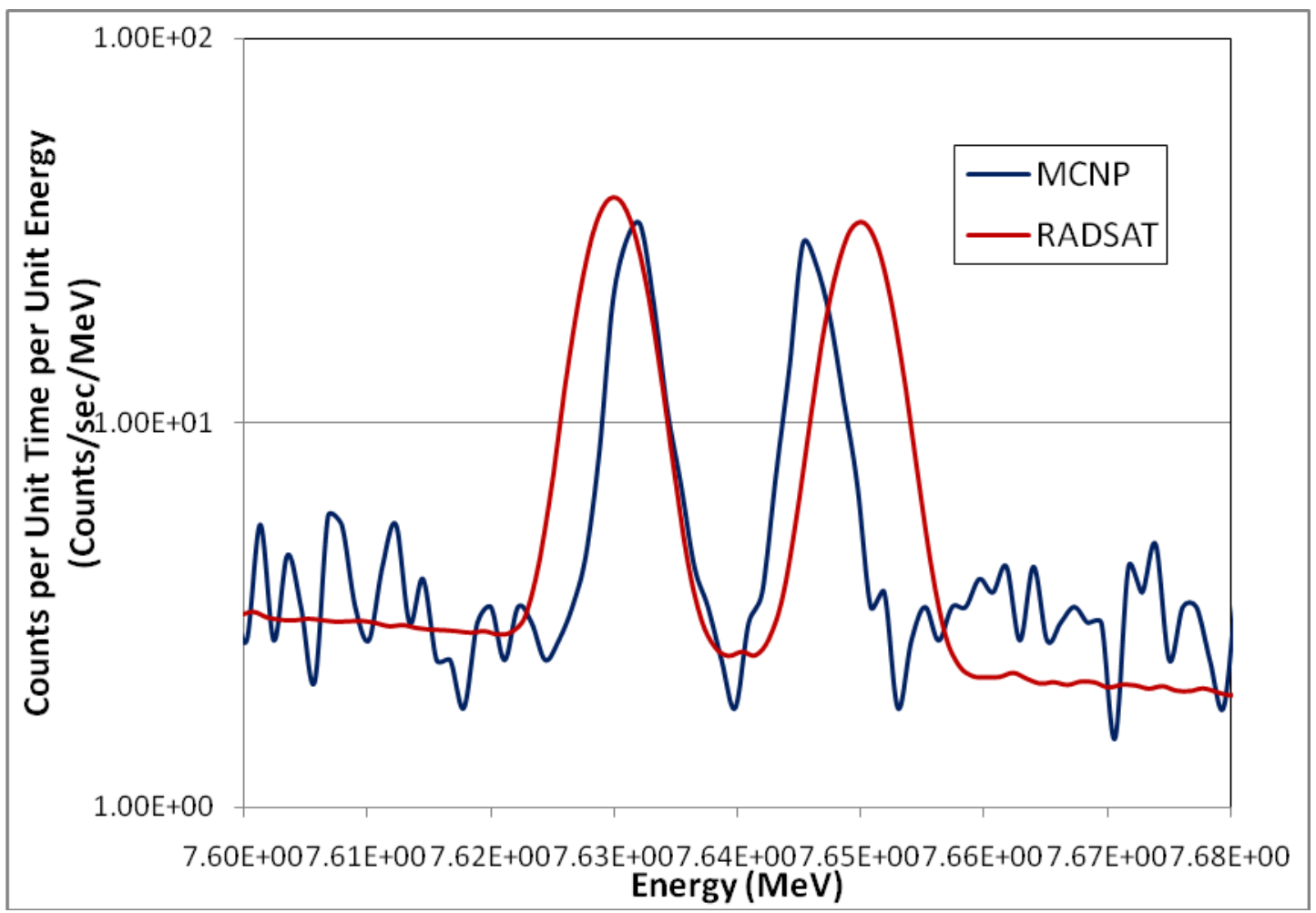

Figure 21. Comparison of 7.631 MeV and 7.645 MeV iron doublet in MCNP and RADSAT detector response for FS irradiated in PINS configuration.

\subsection{Nerve Agent (GB)}

GB is another important chemical weapon material for PINS applications. GB was simulated in a 155mm artillery shell in the typical PINS configuration. The nominal composition of GB was taken to be 7.1\% hydrogen, $22.9 \%$ oxygen, $22.1 \%$ phosphorus, $34.3 \%$ carbon, and $13.6 \%$ fluorine [11] and has a density of 1.102 grams $/ \mathrm{cm}^{3}$ [12]. The experimental results provided by INL for the GB irradiation is shown in Figure 22, and the comparison of the MCNP and RADSAT calculations is shown in Figure 23. Similar to the HD case, the MCNP calculation was completed using the ENDF/B-VII cross section library, while the RADSAT calculation was completed using a modified ENDF/B-VII cross section library. 


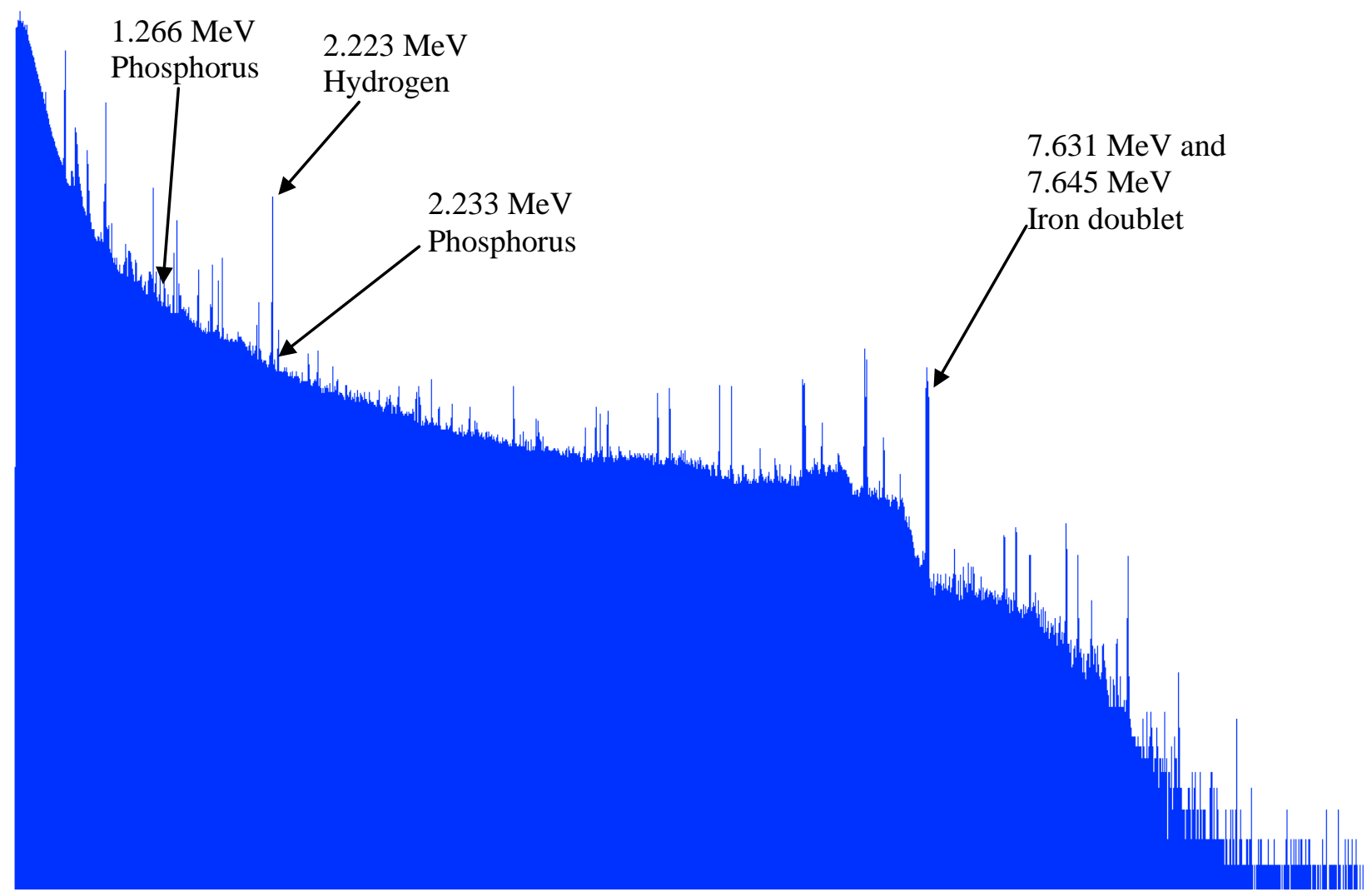

Figure 22. Empirical data from the irradiation of GB (Data provided by INL).

A comparison of the peak count rates for regions of interest is provided in Table 5. Comparisons of the 2.223 MeV hydrogen peak, 1.266 MeV and 2.233 MeV phosphorus peak, 7.631 MeV and 7.645 MeV iron doublet are shown in the following figures (Figures 24 thru 26). Unlike the other munition calculations, the peak heights were not as comparable to the peak heights calculated using MCNP. Also, the continuum in the 4.0 to $8.0 \mathrm{MeV}$ range deviates from that calculated by MCNP. It is believed that the neutron flux used for creating the gamma spectrum may not have been fully converged, which could have produced this deviation in the induced gamma ray spectrum. Additionally, the $2.233 \mathrm{MeV}$ phosphorus peak is absent from the RADSAT graph. RADSAT uses a code, CEPXS, to process the gamma ray cross sections [14]. CEPXS is currently only capable of processing 300 gamma ray groups with 149 discrete gamma rays. Due to the magnitude of the $2.233 \mathrm{MeV}$ phosphorus peak, it was less prominent than 149 other gamma rays in the induced spectrum, causing it not to be carried though as a discrete gamma ray in the RADSAT calculation. In the future, RADSAT should consider expanding the capabilities of CEPXS to be capable of processing more the of 300 gamma ray groups.

Table 5. Peak count rates for regions of interest from the irradiation of GB.

\begin{tabular}{|c|c|c|c|c|c|}
\hline & $1.266 \mathrm{MeV}$ & $2.223 \mathrm{MeV}$ & $2.233 \mathrm{MeV}$ & $7.631 \mathrm{MeV}$ & $7.645 \mathrm{MeV}$ \\
\hline Empirical & 432 & 10139 & 86 & 1014 & 872 \\
\hline MCNP & 158 & 2740 & 64.2 & 354 & 292 \\
\hline RADSAT & 840 & 1040 & 10.5 & 781 & 673 \\
\hline
\end{tabular}




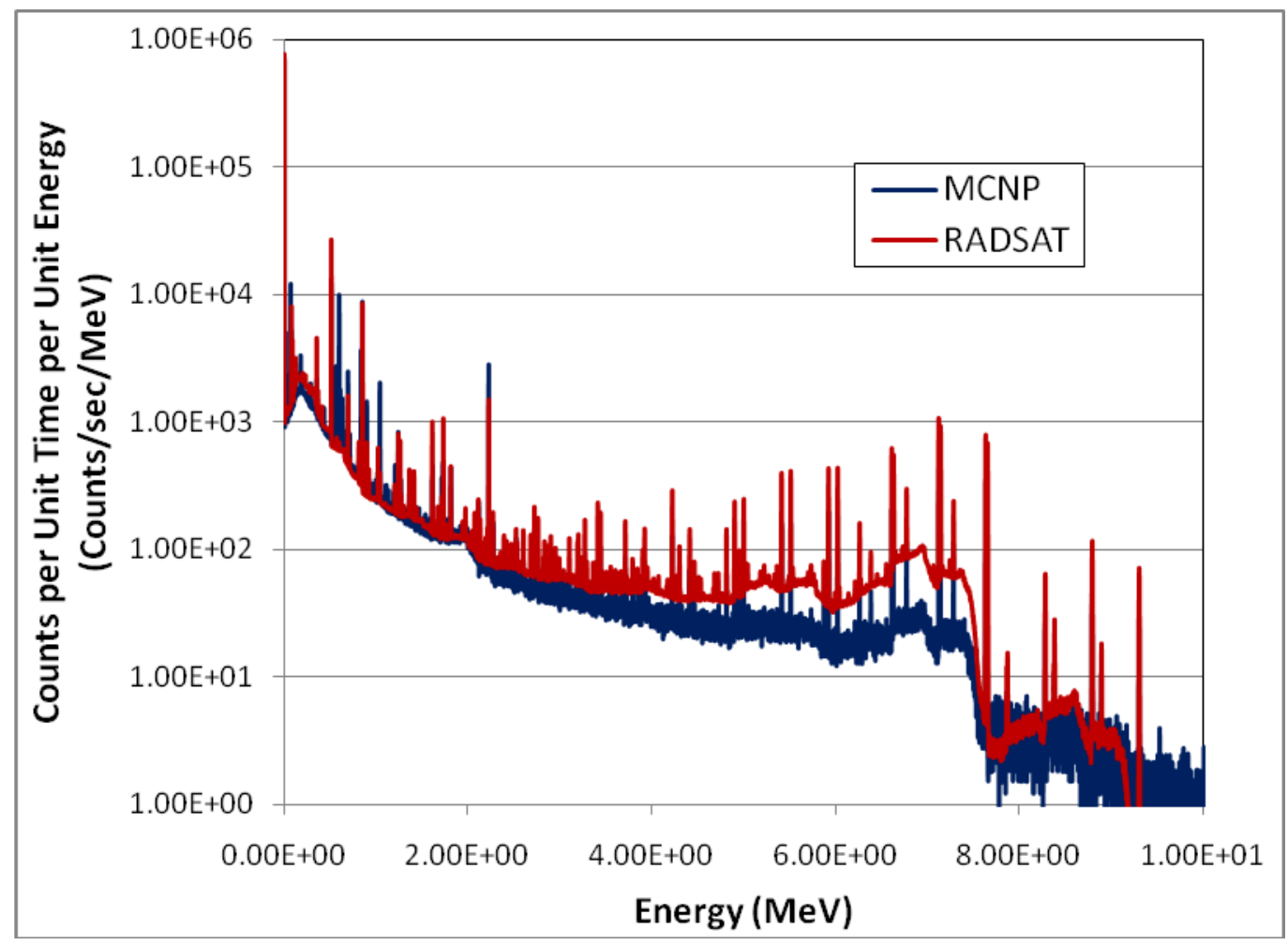

Figure 23. Comparison of MCNP and RADSAT detector response for GB irradiated in PINS configuration.

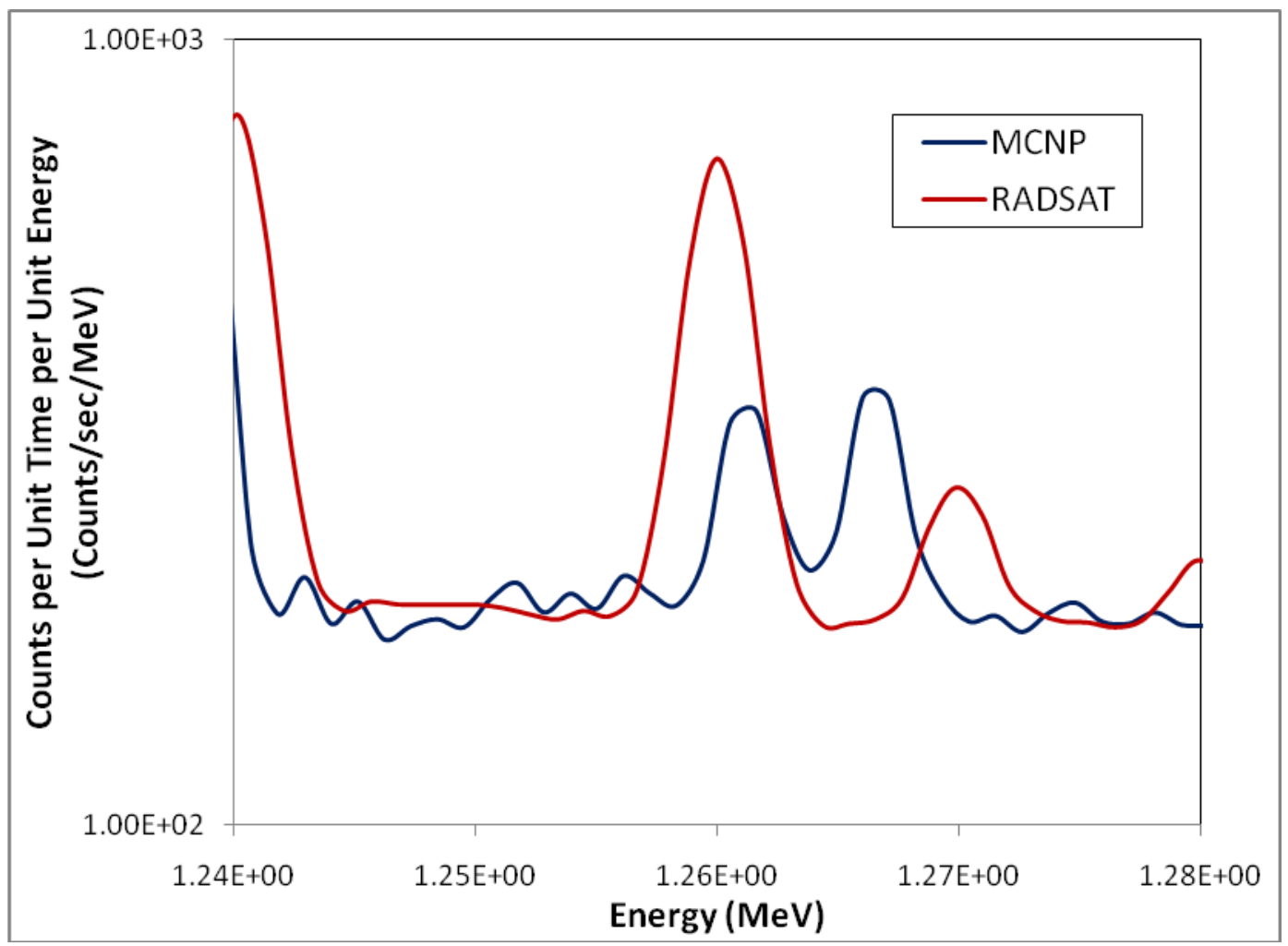

Figure 24. Comparison of 1.266 MeV phosphorus peak in MCNP and RADSAT detector response for GB irradiated in PINS configuration. 


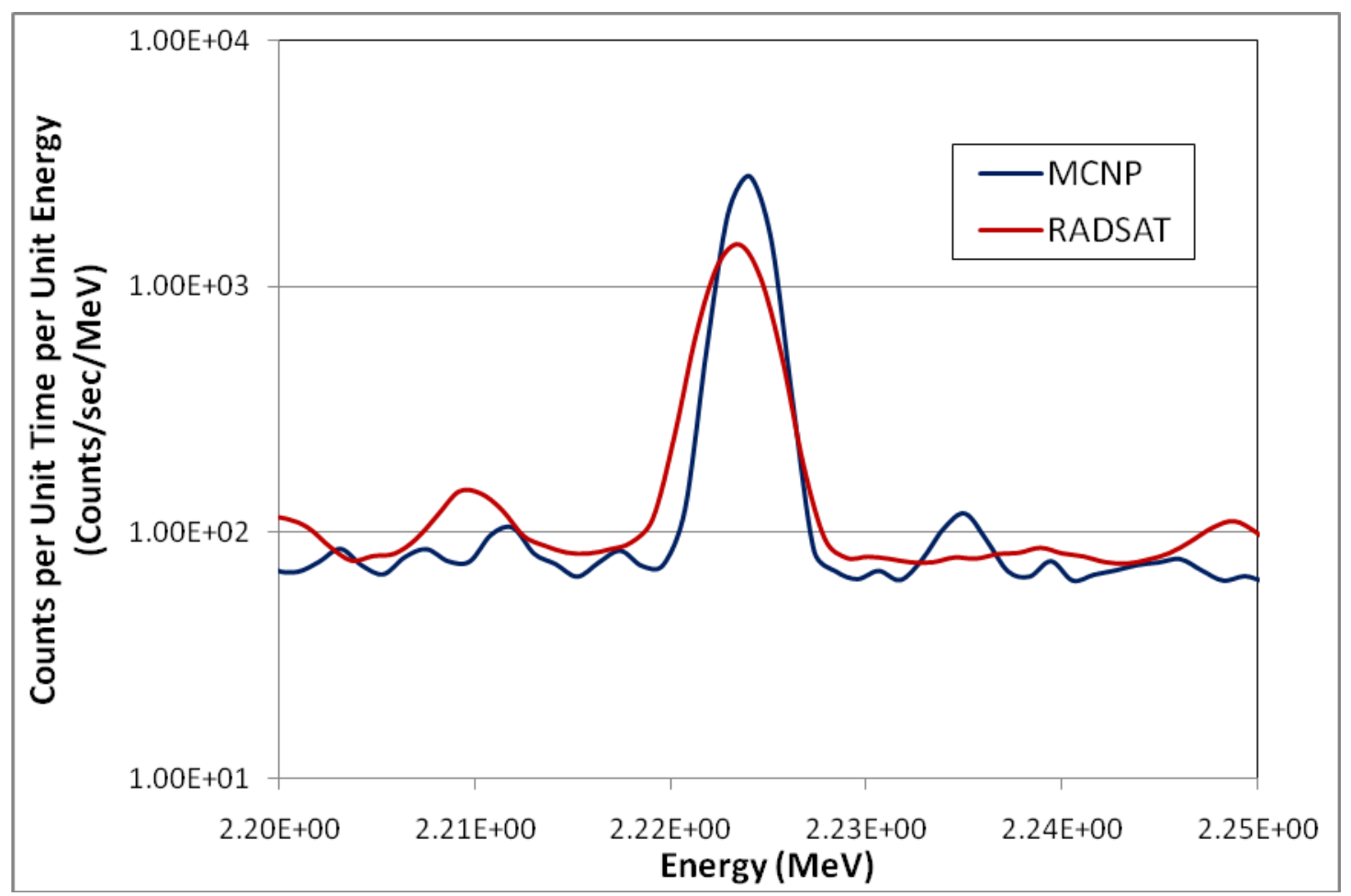

Figure 25. Comparison of 2.223 MeV hydrogen and 2.233 MeV phosphorus peaks in MCNP and RADSAT detector response for GB irradiated in PINS configuration.

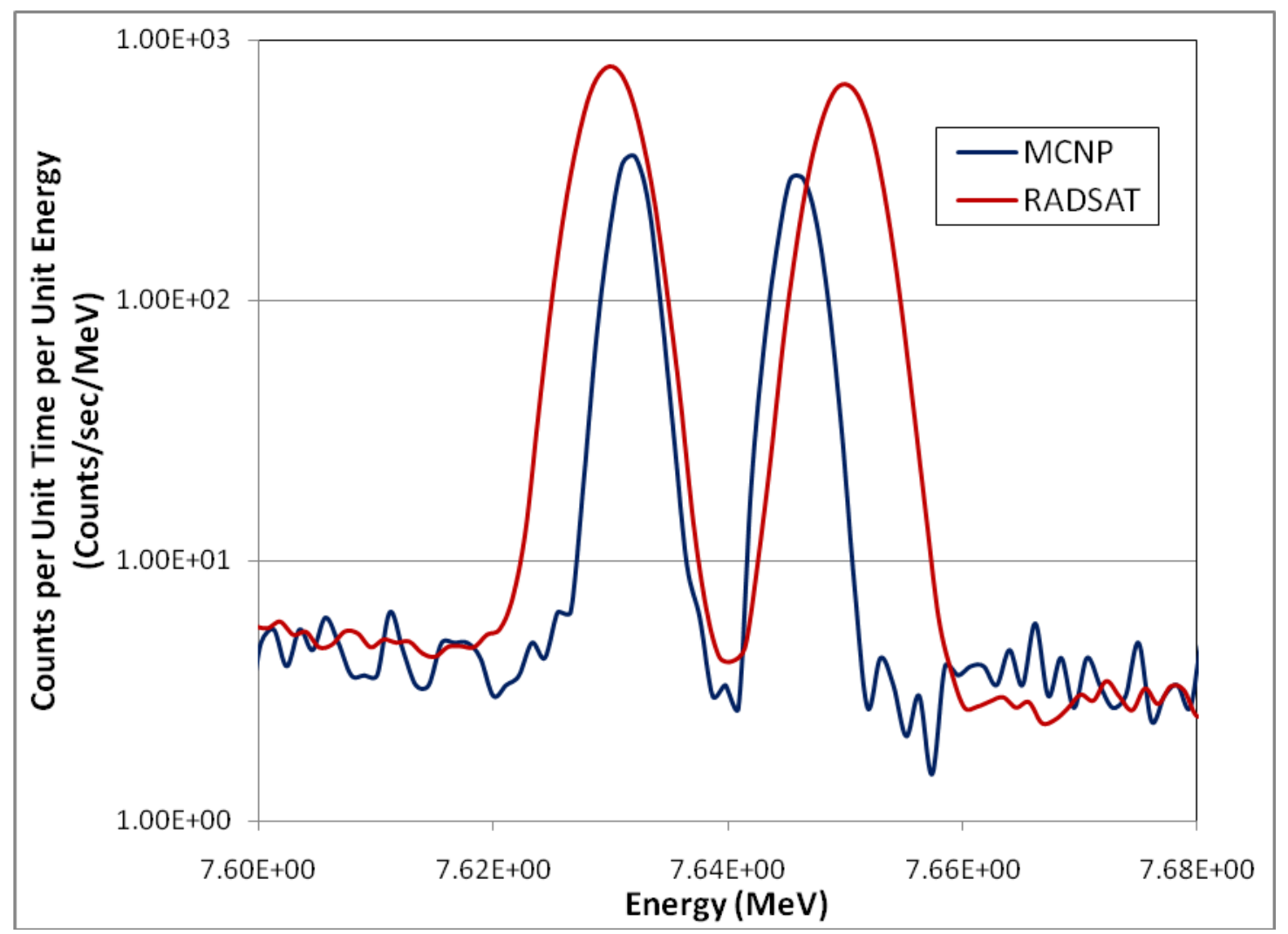

Figure 26. Comparison of 7.631 MeV and 7.645 MeV iron doublet in MCNP and RADSAT detector response for GB irradiated in PINS configuration. 


\subsection{Persistent Nerve Agent (VX)}

Another important chemical weapon material for PINS applications is VX. In RADSAT, VX was simulated in a 155-mm artillery shell in the typical PINS configuration. The nominal VX smoke composition was taken to be $9.7 \%$ hydrogen, $5.2 \%$ hydrogen, $11.6 \%$ phosphorus, $49.4 \%$ carbon, $12.0 \%$ oxygen, and $12.0 \%$ sulfur [11] and has a density of 1.0083 grams $/ \mathrm{cm}^{3}$ [12]. The experimental results provided by INL for the VX irradiation is shown in Figure 27, and the comparison of the MCNP and RADSAT calculations is shown in Figure 28. Similar to the other munition cases, the MCNP calculation was completed using the ENDF/B-VII cross section library, while the RADSAT calculation was completed using a modified ENDF/B-VII cross section library.

A comparison of the count rates in the regions of interest is provided in Table y and, comparisons of the regions of interest for PINS are shown in Figures 29 thru 32 (1.266 MeV sulfur, 2.223 hydrogen, 2.23 sulfur, and 7.631 and $7.645 \mathrm{MeV}$ iron doublet). In both the MCNP and RADSAT spectra, the 2.23 MeV sulfur and 2.233 MeV phosphorus peaks are graphed in the same bin due to limitations in the resolution of the bin widths. The nitrogen peaks were outside of the energy region simulated in the detector response for both MCNP and RADSAT. The remainder of the peak height and energy positioning is comparable to that reported to the spectra in other munition modeling.

Table 6. Peak count rates for regions of interest from the irradiation of VX.

\begin{tabular}{|c|c|c|c|c|c|}
\hline & $1.266 \mathrm{MeV}$ & $2.223 \mathrm{MeV}$ & $2.23 \mathrm{MeV}$ & $7.631 \mathrm{MeV}$ & $7.645 \mathrm{MeV}$ \\
\hline Empirical & 217 & 11920 & 100 & 865 & 795 \\
\hline MCNP & 152 & 3910 & 248 & 350 & 325 \\
\hline RADSAT & 278 & 6100 & 93.0 & 409 & 354
\end{tabular}

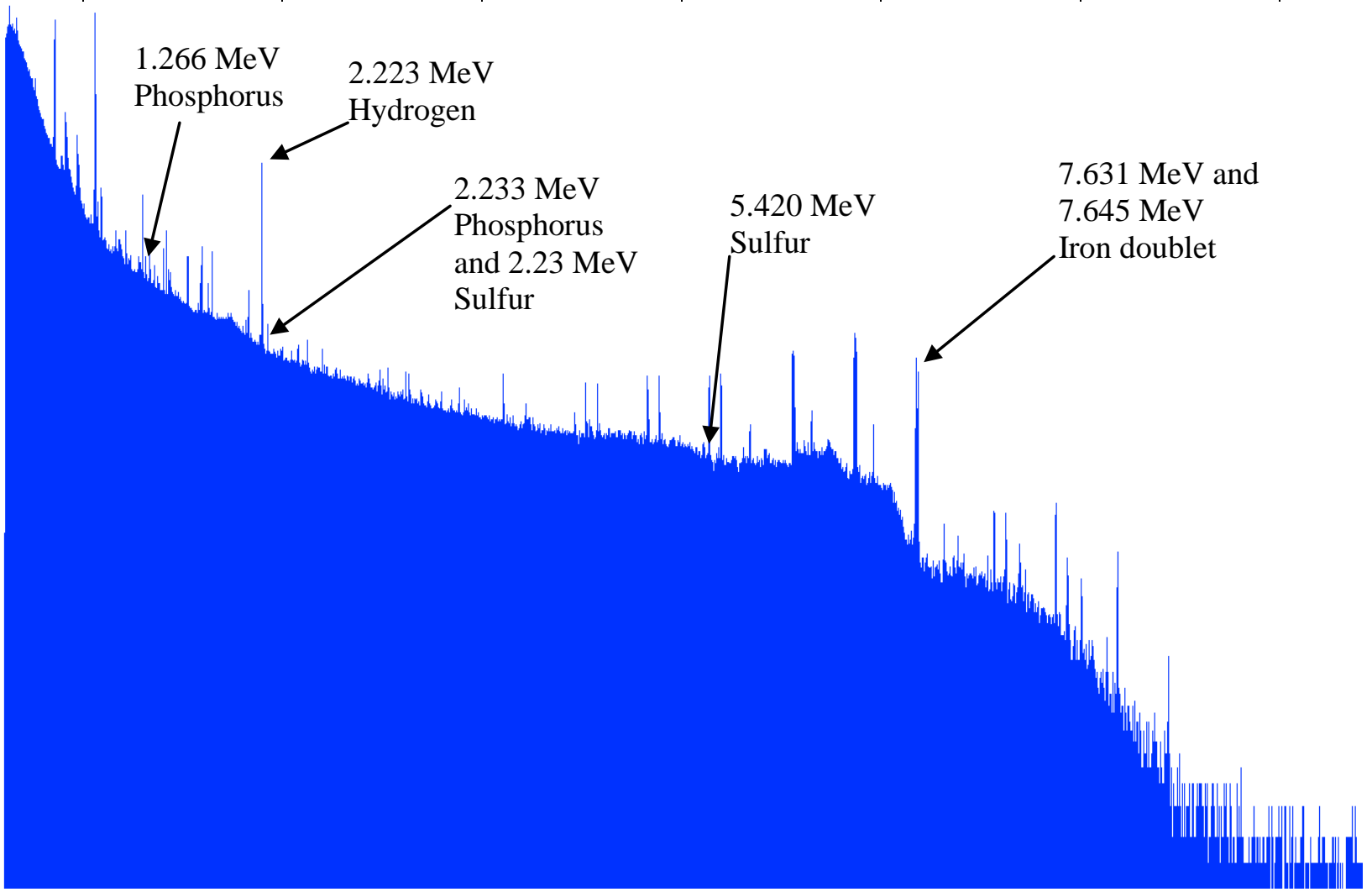

Figure 27. Empirical data from the irradiation of VX (Data provided by INL). 


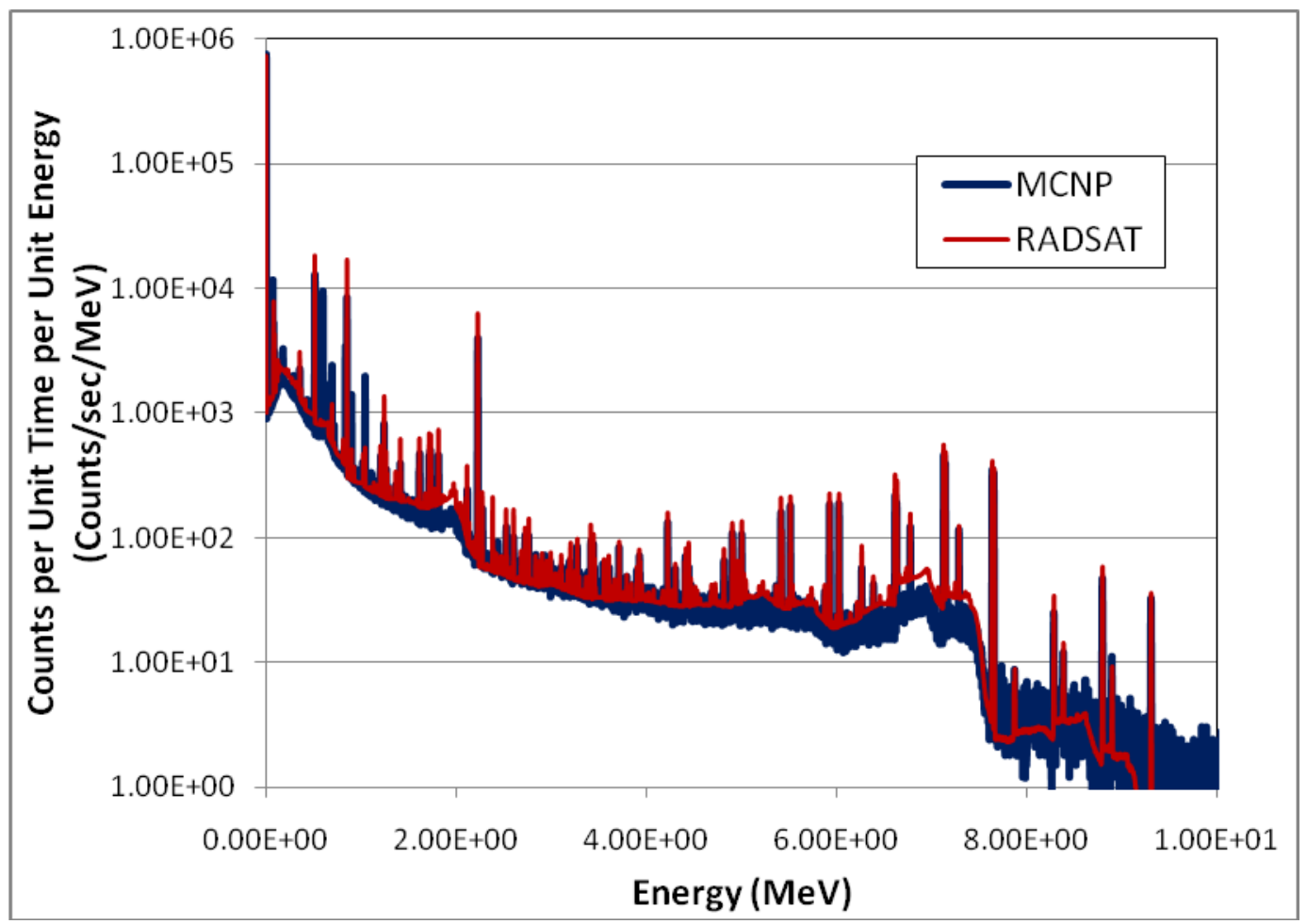

Figure 28. Comparison of MCNP and RADSAT detector response for VX irradiated in PINS configuration.

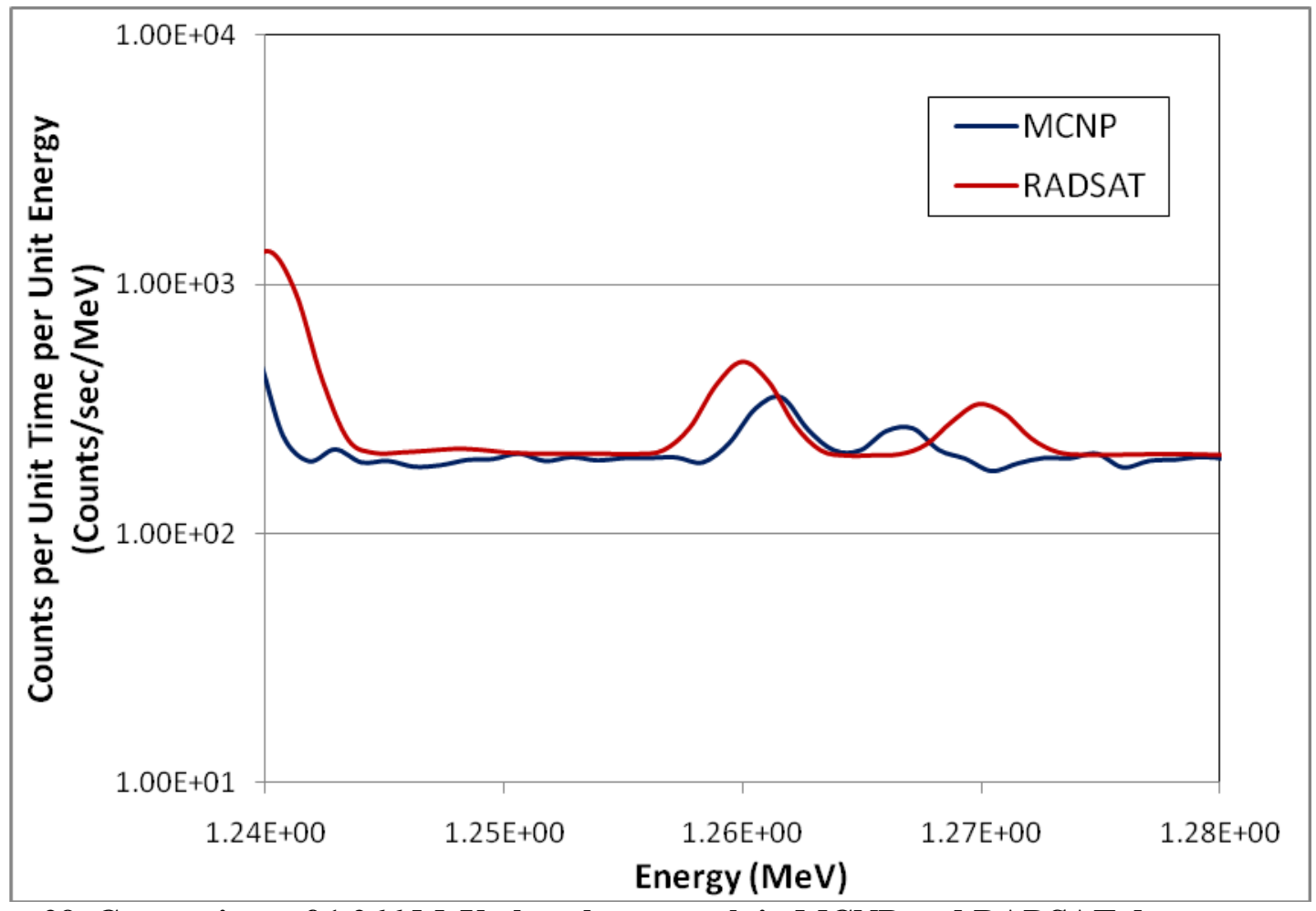

Figure 29. Comparison of 1.266 MeV phosphorus peak in MCNP and RADSAT detector response for VX irradiated in PINS configuration. 


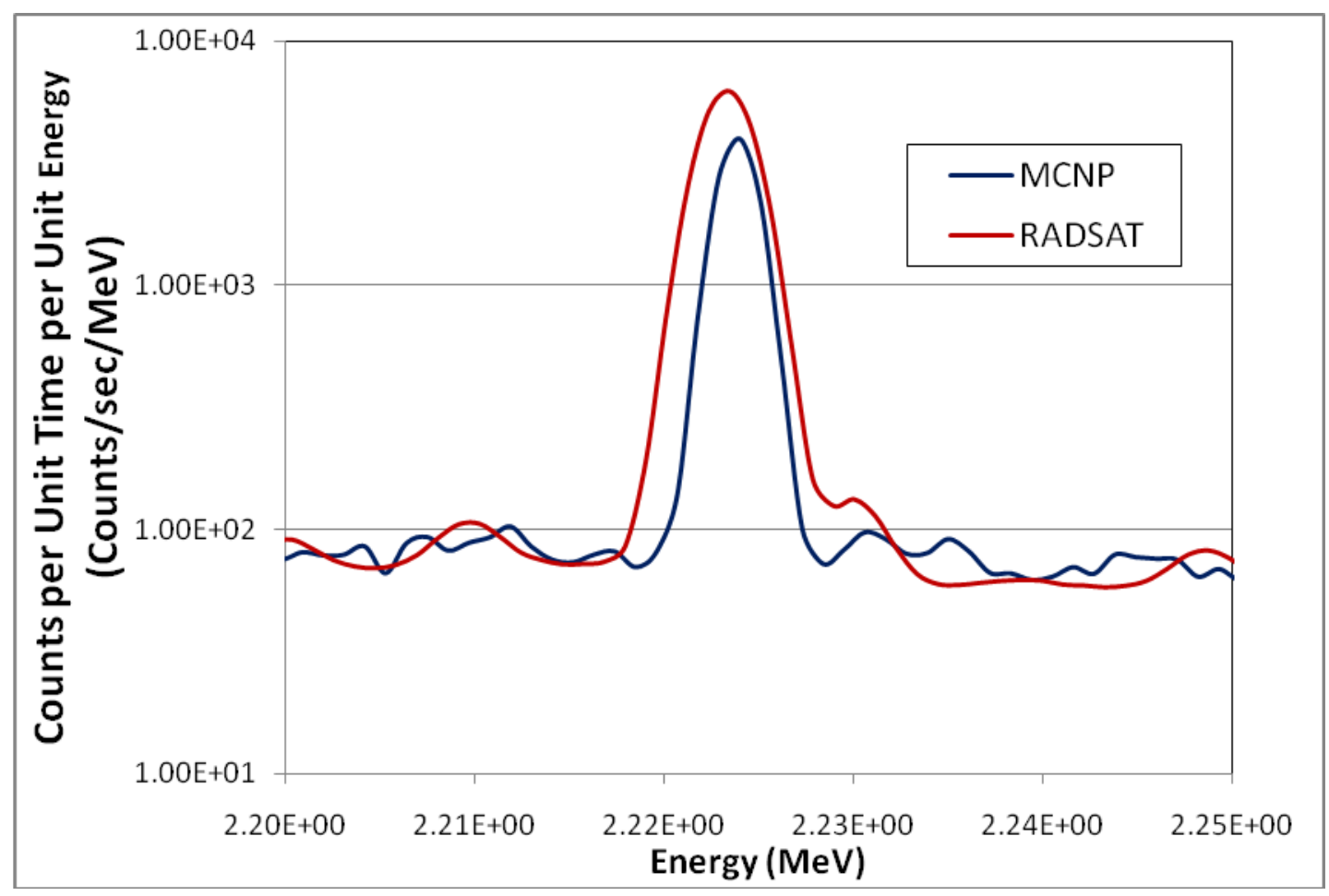

Figure 30. Comparison of 2.223 MeV hydrogen, 2.23 MeV sulfur, and 2.233 MeV phosphorus peaks in MCNP and RADSAT detector response for VX irradiated in PINS configuration.

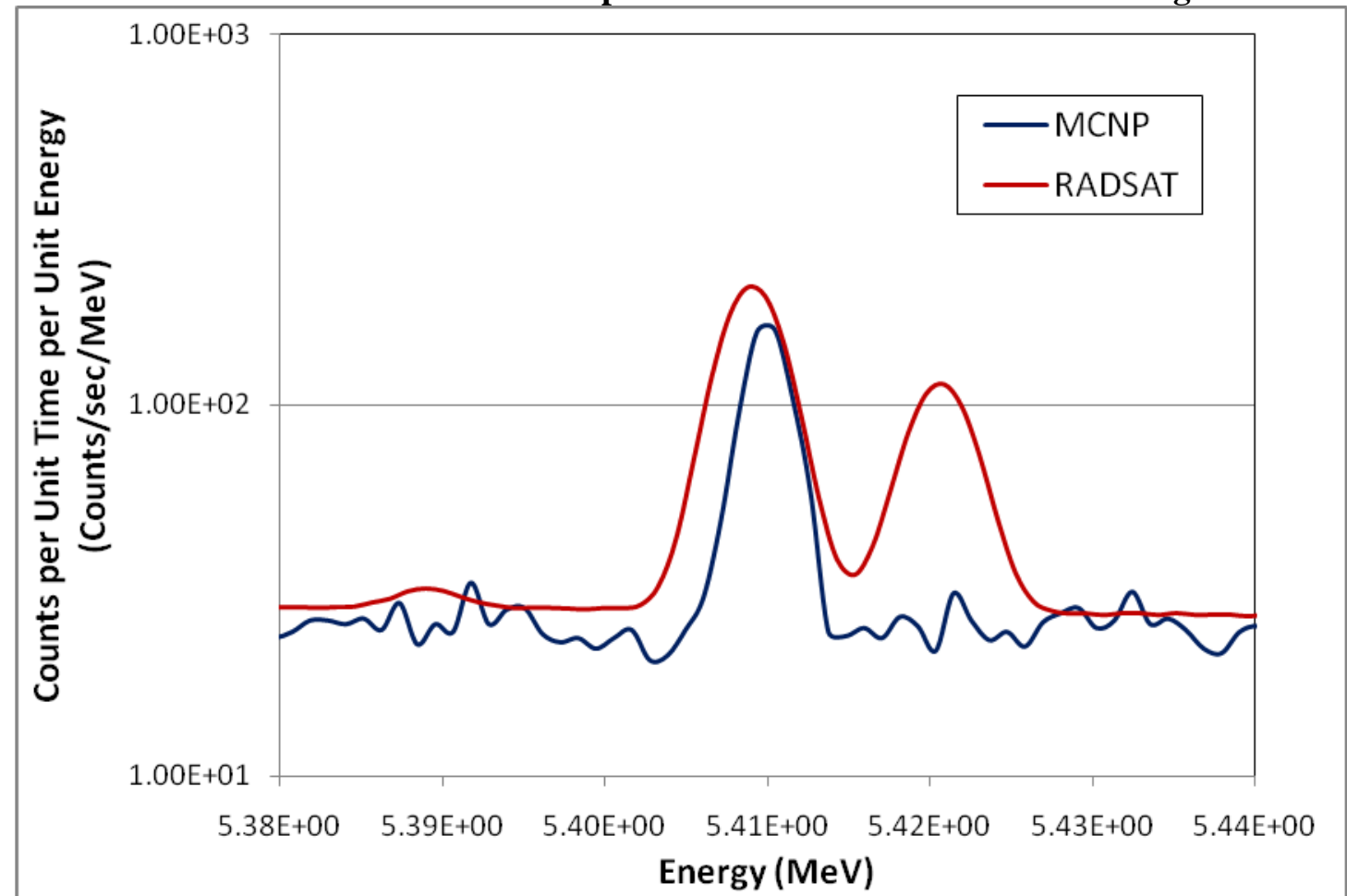

Figure 31. Comparison of 5.421 MeV sulfur peak in MCNP and RADSAT detector response for VX irradiated in PINS configuration. 


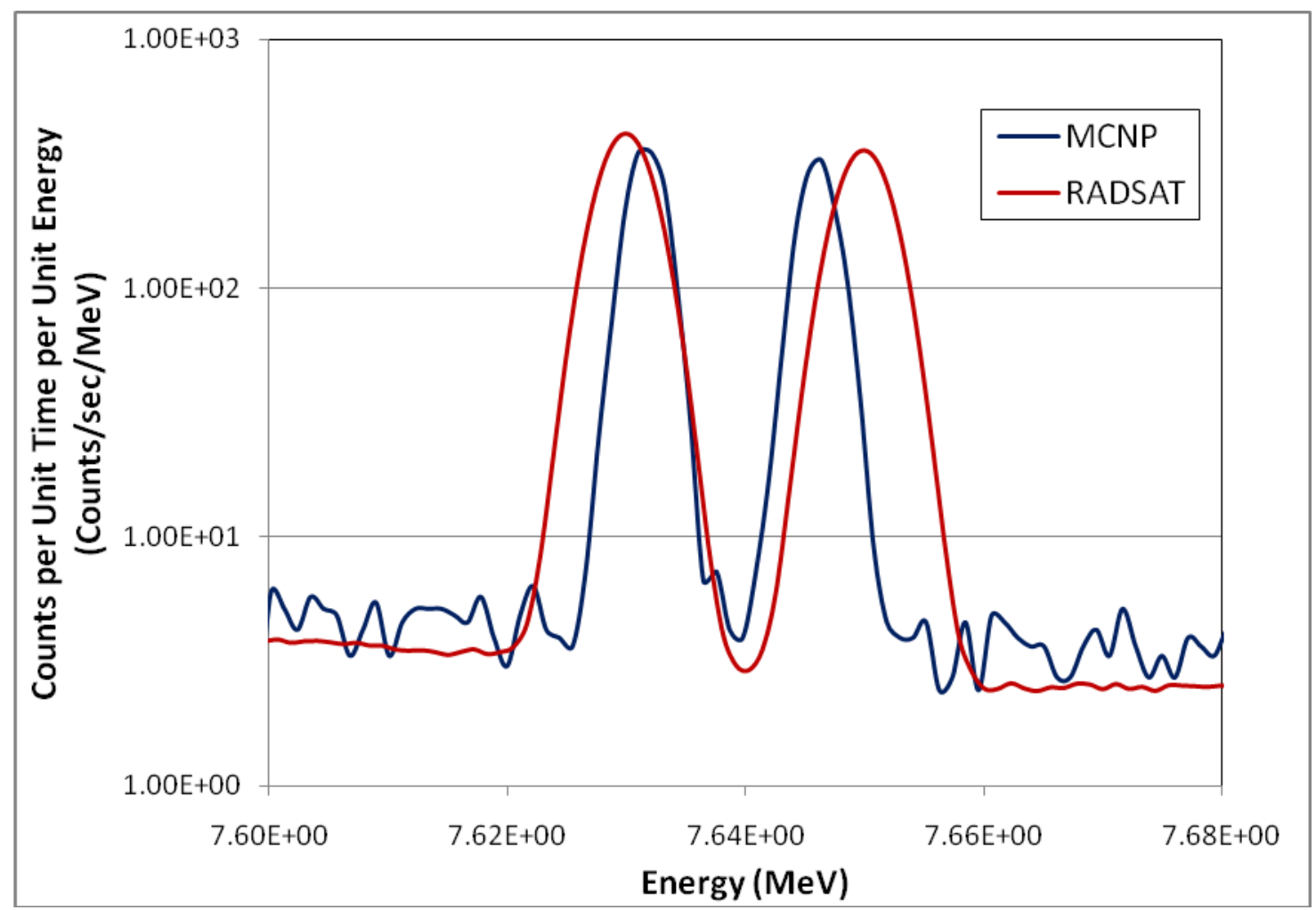

Figure 32. Comparison of 7.631 MeV and 7.645 MeV iron doublet in MCNP and RADSAT detector response for VX irradiated in PINS configuration.

\subsection{Conclusions}

Based on the series of benchmarking problems and comparisons to MCNP5 results, the deterministic transport methods being developed and implemented in RADSAT are capable of accurately and efficiently modeling neutron-activation analysis problems using high-resolution gamma-ray spectrometers for prompt gamma neutron activation analysis. The key enabler for this functionality is the RADSAT-NG multi-group cross section preparation method, which, unlike currently available neutron-gamma cross section libraries and preparation methods, tabulates the discrete-energy and continuum neutron-induced photon source terms separately. These tabulated neutron-gamma cross section libraries allowed for the inclusion of some missing discrete gamma rays in the ENDF/B-VII cross section library which other cross section processing methods are not capable of including. The possibility of contributing more gamma production to a single isotope than naturally occurs by including extra discrete gamma rays that may also be included in the continuum should be understood.

Demonstrating solution accuracy was paramount for these benchmark problems. In the benchmarking process, some deficiencies in the current RADSAT method were discovered for certain problems. Although the RADSAT method proved sufficient for modeling munitions, it was insufficient for modeling simple surrogates (i.e. water or graphite) in the PINS configuration. The gamma ray spectrum that is generated in the remainder of the PINS configuration for these simple surrogates is more complex than the spectrum generated from the surrogate. This discrepancy generates a RADSAT spectrum that cannot be compared to the measured spectrum or MCNP generated spectrum. In order to rectify this discrepancy, it would be necessary to generate the cross sections for compositions in the remainder of the PINS configuration so the total gamma ray spectrum produced in all of the components can be modeled. 
While demonstrating solution accuracy is the necessary first step in the use of RADSAT for neutron activation analysis problems, the primary motivator for using deterministic over Monte Carlo methods is the potential for significantly shorter computational times that might enable new approaches to field analysis of PGNAA spectra recorded from unexploded chemical munitions. Run times were approximately 10 times shorter than benchmark cases described in this report. It is important to note, however, that these run-time comparisons can vary widely depending on the composition of materials in the problem, size of the problem, and skill of the analyst in extracting the best performance from RADSAT and MCNP. In this preliminary work, little effort was made to optimize the RADSAT calculation parameters in order to reduce run time, nor was any substantive variance reduction methods (beyond geometry biasing on the neutron source) applied in the MCNP simulations. Therefore, this work represents only a preliminary assessment of RADSAT methods using a narrow subset of neutronactivation problems. The results should not be interpreted to mean that the RADSAT-NG cross section preparation methods are mature and well vetted or that RADSAT will always offer improved computational efficiency over Monte Carlo methods for neutron-activation problems. Ongoing work, including additional evaluation of spatial discretization in the calculation of neutron flux and neutroninduced photon source terms, will shed more light on both aspects-accuracy and computational efficiency — and allow a more thorough evaluation of the strengths and limitations of RADSAT methods over a wide range of radiation detection problems.

\subsection{Future Work}

Although the initial RADSAT method produces promising results for PGNAA problems, additional work is needed to show that RADSAT is truly useful for real-world applications like the INL PINS project. The highest-priority task is to add the cross sections for the other isotopes present in the problem in order to properly complete all of the provided benchmark problems. Although the graphite, water and sulfur surrogates were modeled, the lack of cross sections for the remaining compositions caused major discrepancies in the calculated detector response. Small differences can be noted in the MCNP and RADSAT results for the munition simulation also which can be attributed to the assumption that only the munition produces gamma rays.

The chemical composition and munition-type library should be expanded for PGNAA problems using RADSAT. Currently, HD, FS, GB and VX have been simulated for three configurations, the 4.2-inch mortar, $155 \mathrm{~mm}$ mortar and the Livens munition. Adding chemical compositions that rely on other peak ratios for identifications may also identify other areas in need of improvement and expand the required RADSAT-NG cross section library. For example, INL has previously indicated interest in the modeling WP smoke.

Identifying ways to reduce RADSAT computational time for PGNAA problems is another important improvement. To date, calculations were completed in parallel with preliminary methods development, and little effort was made to optimize the transport parameters (i.e., mesh size, $\mathrm{P}_{\mathrm{N}}$ order, $\mathrm{S}_{\mathrm{N}}$ order) to reduce computational times. Since a standard geometric configuration is used for PINS problems (i.e. source to target to detector orientation and position) with a limited type of munitions, the transport parameters can be optimized for that specific geometry, thereby reducing computational time.

Using the alpha version of RADSAT, the neutron transport calculation in PINS-like problems accounts for over $90 \%$ of the computation time. Since the PINS measurements are primarily conducted on a given range of material compositions and munition configurations, it may be possible to pre-calculate a base set of neutron spectra that will cover the range of neutron spectra seen within chemical munitions in PGNAA 
problems. These spectra would be stored in a database for use as an input for calculating the neutroninduced photon spectrum. If this is possible, only the photon transport calculation need be completed during the iterative process, significantly reducing the total computational time for a problem (e.g., by a factor of 10). A set of trials to determine the relative sensitivity of the neutron-induced photon spectrum on the initial neutron flux will help determine the viability of this method.

In addition to the possibility of eliminating the neutron-transport calculation from the iterative process, it may be possible to reduce the time required to complete the photon-transport calculation. PGNAA problems are typically interested in net peak count rates or peak ratios, not the entire photon spectrum. Yet at present, much of the photon-transport computational time is spent calculating the scattered component of the flux. Since the uncollided component is the primary data of interest for PGNAA problems, it is likely that a ray-tracing calculation only would be adequate. This eliminates the timeconsuming calculation of the scattered component from the photon-transport calculation. This would also provide the all-important net peak counts for full-energy peaks and will simultaneously reduce the amount of time required to complete the calculation.

Once it has been determined that the RADSAT method is accurately capturing PGNAA problems, those methods can be implemented in the RADSAT graphical user interface or some alternative version of that user interface tailored for the specific applications (e.g., PINS analysis in the field).

\subsection{References}

1. Smith, L.E. et al., "Coupling Deterministic and Monte Carlo Transport Methods for the Simulation of Gamma-Ray Spectroscopy Scenarios,” IEEE Trans. Nucl. Sci. (2008).

2. McGhee, J.M. et al., Attila ${ }^{\circledR}$ User’s Manual, Transpire, Inc. (2007).

3. Shaver, M.W. et al., "The Coupling of a Deterministic Transport Field Solution to a Monte Carlo Boundary Condition for the Simulation of Large Gamma-Ray Spectrometers,” Nuclear Technology Vol. 168, pp. 95-100 (2009).

4. Burns, K.A., "Coupled Multi-Group Neutron Photon Transport for the Simulation of High-Resolution Gamma-Ray Spectroscopy Applications” Georgia Institute of Technology (2009).

5. Burns, K.A. et. al., "Multi-Group Transport Methods for High-Resolution Neutron Activation Analysis,” submitted to Nuclear Technology (2011).

6. MCNP-A General Monte Carlo N-Particle Transport Code, Los Alamos National Laboratory, LAUR-03-1987 (2003).

7. Varley, E.S. and J. Mattingly, "Rapid Feynman-Y Synthesis: Kynea3 Cross section Library Development,” Trans. Amer. Nucl. Soc., Vol. 98, pp.575-576 (2008).

8. NJOY99.0 - Code System for Producing Pointwise and Multi-group Neutron and Photon Cross sections from ENDF/B Data, Oak Ridge National Laboratory, PSR-480/NJOY99.0 (2000).

9. Lorence, L.J., “CEPXS/ONELD Version 2.0: A Discrete Ordinates Code Package for General OneDimensional Coupled Electron-Photon Transport,” IEEE Trans. Nucl. Sci., 39, 1031 (1992).

10. Duderstadt, J.J. and L.J. Hamilton, Nuclear Reactor Analysis, John Wiley \& Sons, Inc., New York, USA (1976).

11. Siedenstrang, A.L. et al., PINS Chemical Assay System, Vol. 4.6, Idaho National Laboratory (2008). 
12. Potential Military Chemical/Biological Agents and Compounds, U.S Army Field Manual FM 3-9, (1990).

13. Military Chemistry and Chemical Compounds, U.S. Army Field Manual FM 3-9, (1975).

14. Lorence, L.J., “CEPXS/ONELD Version 2.0: A Discrete Ordinates Code Package for General OneDimensional Coupled Electron-Photon Transport,” IEEE Trans. Nucl. Sci., 39, 1031, (1992).

15. Hale, G.M., “ENDF/B-VII Material 125 [H-1]”, Las Alamos National Laboratory, (2005).

16. Frankle, S.C. and P.G. Young, “ENDF/B-VI Material 125 [H-1]”, Rev 5, Las Alamos National Laboratory, (1998). 